\title{
Evaluating Stability of Aqueous Multiwalled Carbon Nanotube Nanofluids by Using Different Stabilizers
}

\author{
Tun-Ping Teng, Yan-Bo Fang, Yu-Chun Hsu, and Li Lin \\ Department of Industrial Education, National Taiwan Normal University, No. 162, Sec. 1, He-ping E. Road, Da-an District, \\ Taipei City 10610, Taiwan \\ Correspondence should be addressed to Tun-Ping Teng; tube5711@ntnu.edu.tw
}

Received 13 June 2014; Revised 25 September 2014; Accepted 9 October 2014; Published 10 November 2014

Academic Editor: Margarida Amaral

Copyright (C) 2014 Tun-Ping Teng et al. This is an open access article distributed under the Creative Commons Attribution License, which permits unrestricted use, distribution, and reproduction in any medium, provided the original work is properly cited.

The 0.5 wt.\% multiwalled carbon nanotubes/water nanofluids (MWNFs) were produced by using a two-step synthetic method with different types and concentrations of stabilizers. The static position method, centrifugal sedimentation method, zeta potential measurements, and rheological experiments were used to assess the stability of the MWNFs and to determine the optimal type and fixed MWCNTs-stabilizer concentration of stabilizer. Finally, MWNFs with different concentrations of MWCNTs were produced using the optimal type and fixed concentration ratio of stabilizer, and their stability, thermal conductivity, and $\mathrm{pH}$ were measured to assess the feasibility of using them in heat transfer applications. MWNFs containing SDS and SDBS with MWCNTsstabilizer concentration ratio were $5: 2$ and $5: 4$, respectively, showed excellent stability when they were evaluated by static position, centrifugal sedimentation, zeta potential, and rheological experiments at the same time. The thermal conductivity of the MWNFs indicated that the most suitable dispersing MWNF contained SDBS. MWNFs with MWCNTs concentrations of 0.25 , 0.5, and 1.0 wt.\% were fabricated using an aqueous SDBS solution. In addition, the thermal conductivity of the MWNFs was found to have increased, and the thermal conductivity values were greater than that of water at $25^{\circ} \mathrm{C}$ by $3.20 \%, 8.46 \%$, and $12.49 \%$.

\section{Introduction}

Research on the use of nanofluids in heat transfer applications has been highly productive. Relevant studies pertained to nanofluids having high thermal conductivity, heat transfer coefficient or capacity, viscosity, pumping power, and pressure loss or pipeline friction factor [1-4]. However, the stability of nanofluids is the most crucial indicator for assessing the practicality of using them in heat exchange systems in the long term. The settling of nanoparticles clogs pipelines and can cause damage to the heat exchange system. Improving the stability of nanofluids mainly consists of mechanical stirring, ultrasonic vibration, adjustment of the $\mathrm{pH}$, and adding stabilizer.

The preparation of nanofluids containing different materials, dispersing methods, and stabilizers/pH control is summarized in Table 1. Various mechanical stirring and ultrasonic vibration methods have been commonly used to disperse and break agglomerated nanoparticles for enhancing the stability of nanofluids. Dispersion equipment has its advantages and disadvantages. In addition to the dispersion equipment characteristics, which can affect the stability of nanofluids, the properties of the interface between the nanoparticles and the base fluid are also a key factor affecting stability. For most nanofluids, the use of mechanical and vibration dispersion methods results in high stability for only a short period. Adjusting the $\mathrm{pH}$ and adding various dispersants and surfactants (stabilizers) can enhance the stability of nanofluids. Therefore, different mechanical stirring coordinates are used by adjusting the $\mathrm{pH}$ and adding a stabilizer to improve substantially the long-term stability of nanofluids. The stabilizers can change the characteristics of the interface between the nanoparticles and base liquid and can effectively reduce the agglomeration of nanoparticles in nanofluids, to achieve long-term stable suspension. However, the dispersion effects and thermophysical properties of various types and concentrations of stabilizers depend on the nanoparticles and base fluid $[5,6]$.

The static position method is the most commonly used method to evaluate the stability of nanofluids. The static 


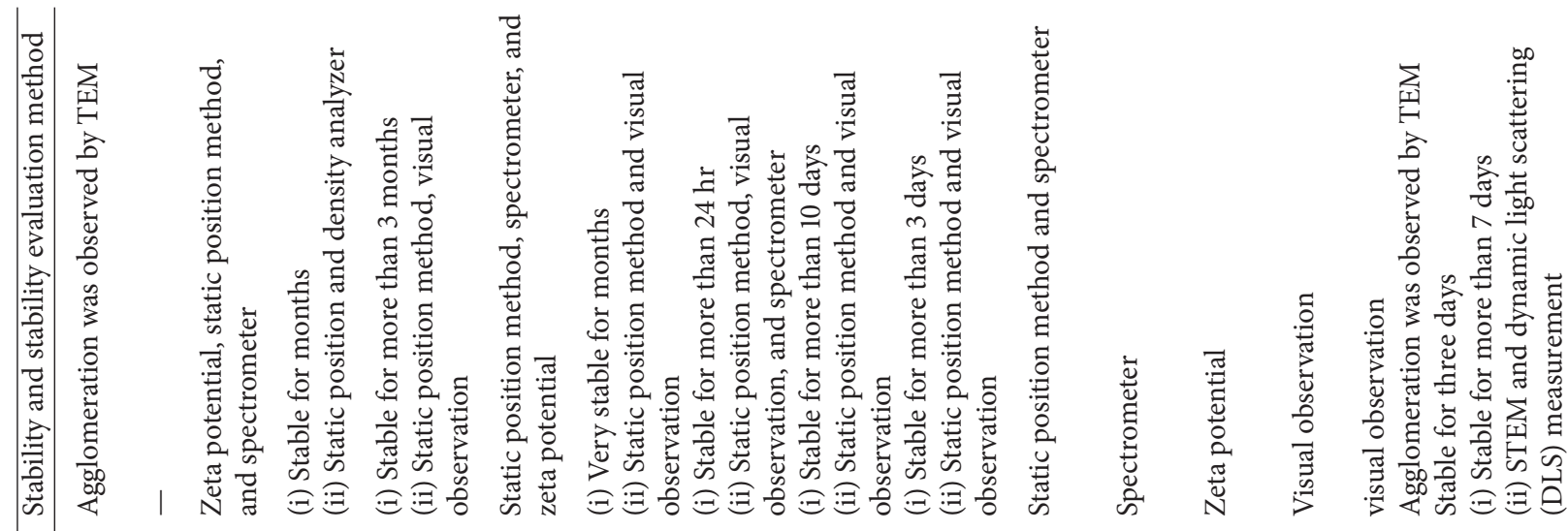

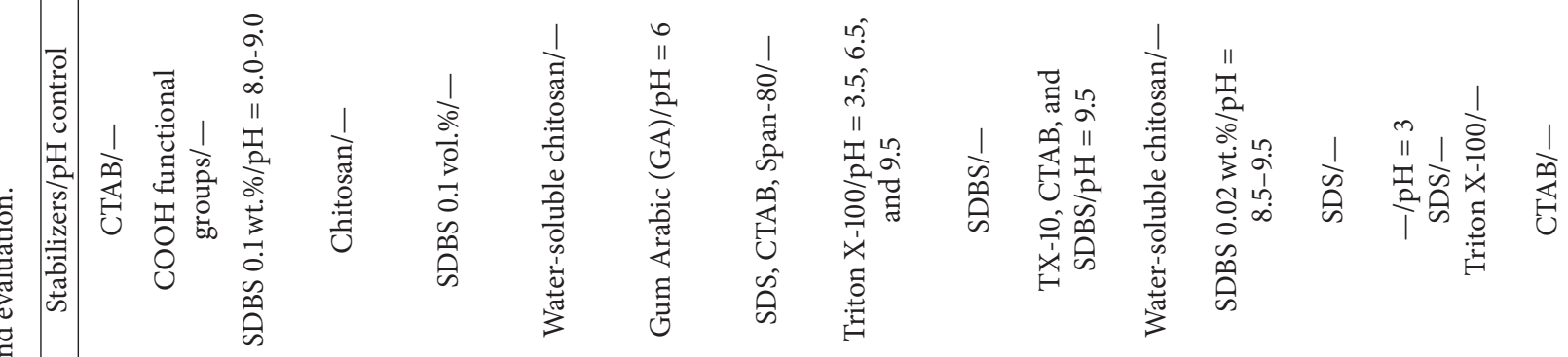

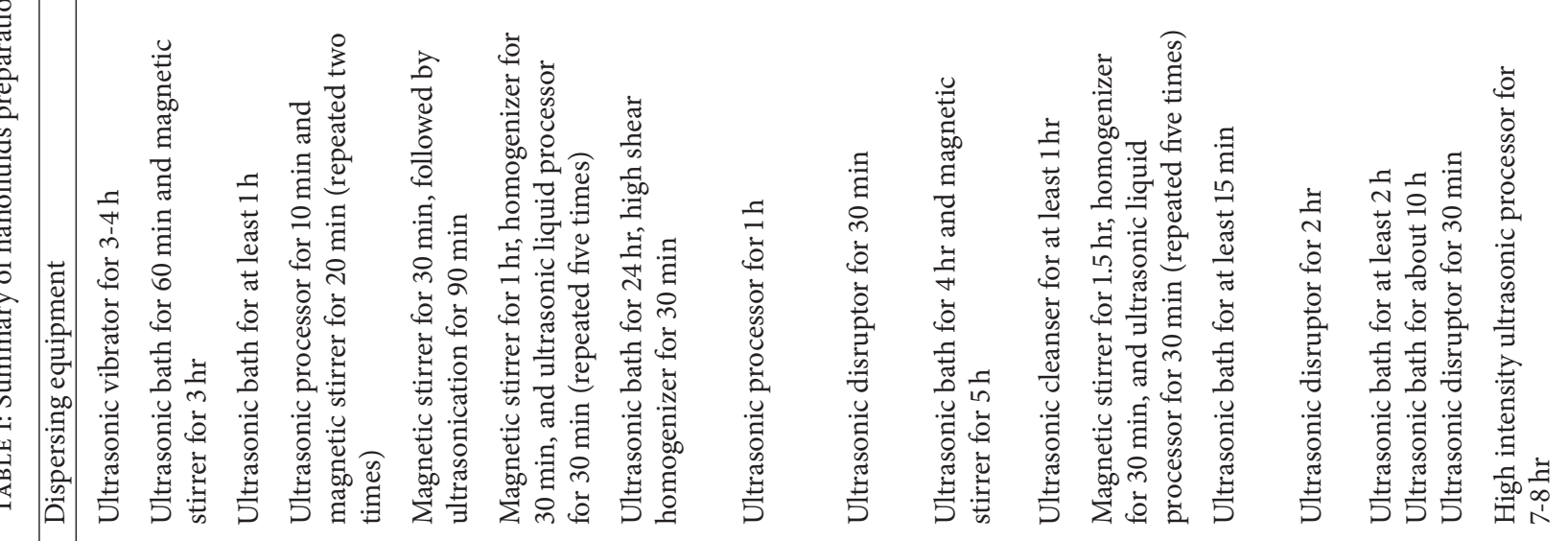

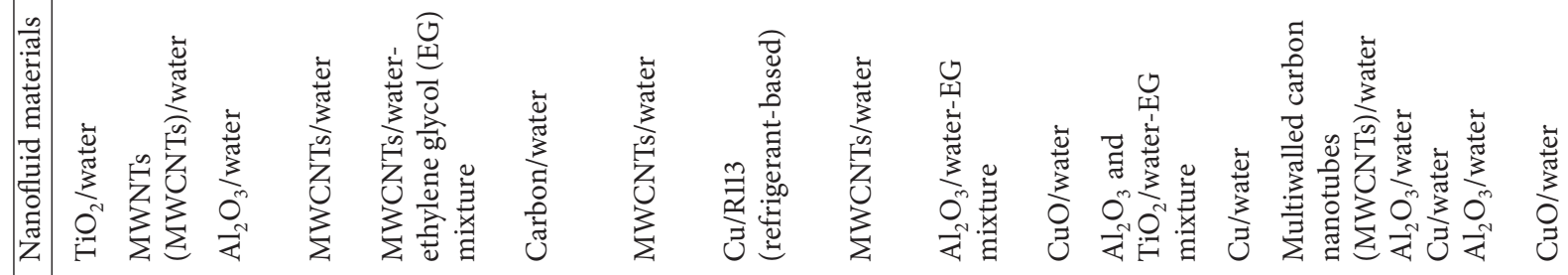

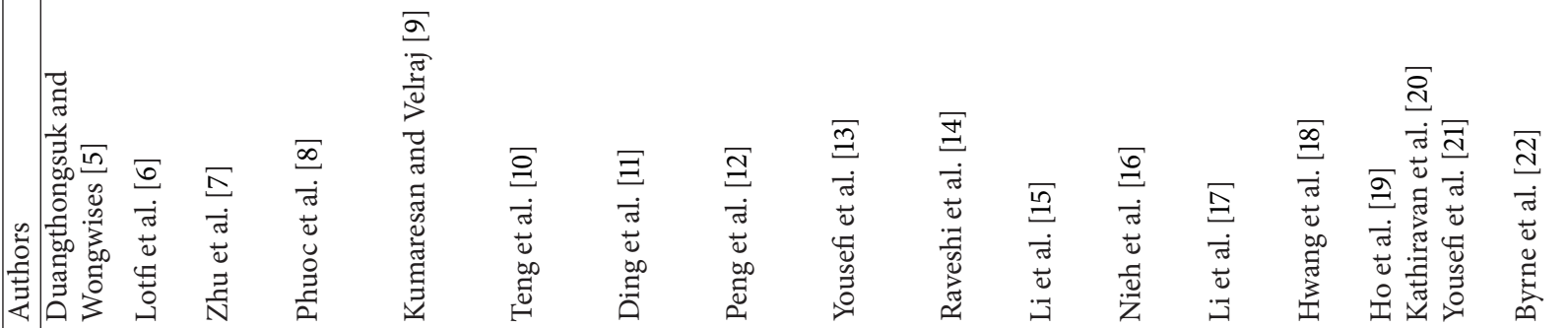




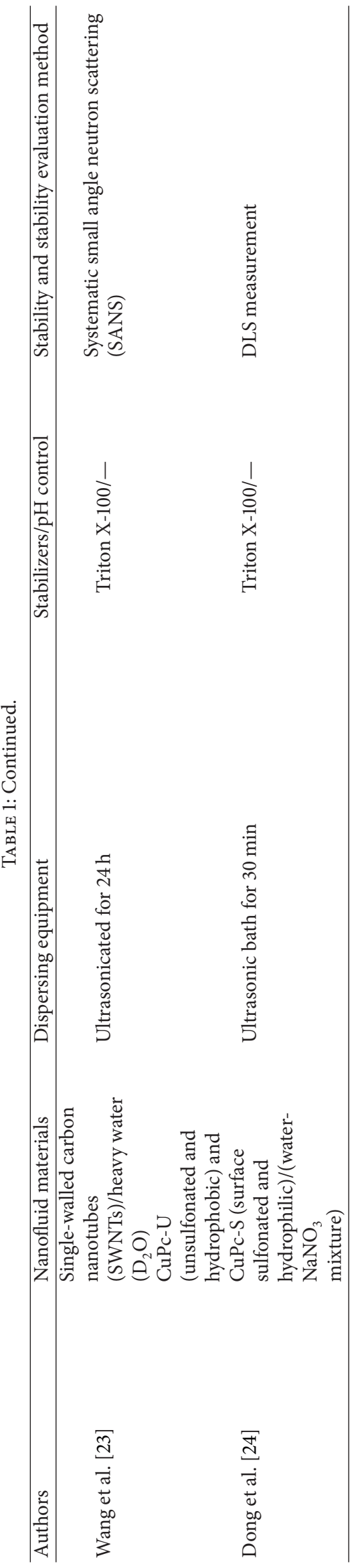


position method leaves nanofluids in containers standing for a particular period, and the distance or color difference in sedimentation between nanofluids was observed by the naked eye [7-15]. The serious sedimentation phenomenon of nanofluids represents poor stability of nanofluids and reduces the applicability of nanofluids. However, this evaluation method cannot accurately determine differences in the stability of nanofluids when the differences in suspension performance and transmittance between nanofluids are small. Therefore, many researchers use a spectrometer to detect transmittance or absorbance differences between nanofluids before and after leaving them to stand, to determine the difference in stability between nanofluids $[7,10,12,15,16]$. However, using a spectrometer to determine the stability of nanofluids continues to be constrained by the transmittance of nanofluids.

The zeta potential $\left(V_{z}\right)$ is also commonly used to evaluate the stability of nanofluids $[7,10,17]$. The $V_{z}$ is the potential difference between the dispersion medium and the stationary layer of fluid attached to the dispersed particle. The magnitude of the $V_{z}$ indicates the degree of electrostatic repulsion between adjacent, similarly charged particles in dispersion. A high $V_{z}$ will confer stability to resist aggregation of particles. When the $V_{z}$ is small, attractive forces may exceed this repulsion and the particles in dispersion may aggregate. Therefore, colloids with high $V_{z}$ (negative or positive) are electrically stabilized, while colloids with low $V_{z}$ (approaching zero) tend to aggregate. The $V_{z}$ value of nanofluids is within the range of \pm 40 to $\pm 60 \mathrm{mV}$, denoting that nanofluids demonstrate favorable stability. When $V_{z}$ of nanofluids is greater than $\pm 60 \mathrm{mV}$, the nanofluids exhibit excellent stability $[25,26]$.

Nanofluids may show solid-liquid separation in the flow state because of the density difference between the nanoparticles and the base liquid, thereby affecting the stability of the nanofluids. Therefore, a dynamic state can be applied to evaluate the stability of nanofluids, which in turn indicates the suitability of nanofluids for use in heat exchange applications in the long term. The preparation and evaluation of nanofluids is summarized in Table 1.

Multiwalled carbon nanotubes (MWCNTs)/water nanofluids (MWNFs) with MWCNTs concentration of $0.5 \mathrm{wt} . \%$ and different stabilizers and stabilizer concentrations were produced using a two-step synthetic method. The static position method, centrifugal sedimentation method, $V_{z}$ measurements, and rheological experiments were used to assess the stability of MWNFs in static and dynamic states and to determine the optimal type and concentration of stabilizer. Finally, MWNFs of different MWCNTs concentrations were produced using the optimal type and concentration ratio of stabilizers, and the stability, thermal conductivity, and $\mathrm{pH}$ of the MWNFs were measured to confirm the usefulness of the selected stabilizer for heat transfer applications.

\section{Preparing MWNFs}

MWCNTs (Cheap Tubes Inc., USA) were used as nanoadditives to prepare the MWNFs. The external diameter,

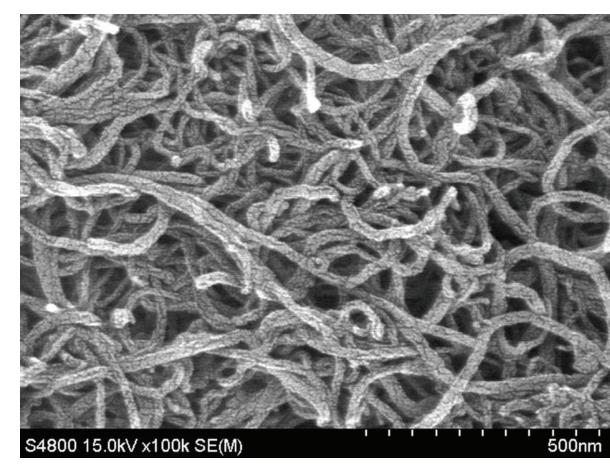

Figure 1: FESEM images of MWCNTs.

internal diameter, length, true density, specific surface area, and purity were approximately $20-30 \mathrm{~nm}, 5-10 \mathrm{~nm}, 10-$ $30 \mu \mathrm{m}, 2100 \mathrm{~kg} / \mathrm{m}^{3}, 110 \mathrm{~m}^{2} / \mathrm{g}$, and $>95 \mathrm{wt} \%$, respectively [27]. Figure 1 shows high-resolution field-emission scanning electron microscopic (HR-FESEM, S4800, Hitachi, Japan) images of the MWCNTs, which appear to be aggregated; the external diameter agrees with the approximate specifications provided by the supplier and the values presented in previous studies $[8,27-29]$.

The MWNFs were produced using a two-step synthetic method. First, an aqueous solution of the stabilizer was prepared by adding $1.6 \mathrm{wt} . \%$ of water-soluble chitosan $\left(\mathrm{CH},\left(\mathrm{C}_{6} \mathrm{H}_{11} \mathrm{O}_{4} \mathrm{~N}\right)_{n}, n=2-20\right.$, C06, Charming \& Beauty, Taiwan), alginic acid sodium salt $\left(\mathrm{AG}, \mathrm{NaC}_{6} \mathrm{H}_{7} \mathrm{O}_{6}\right.$, Alfa Aesar, USA), Triton X-100 (TX100, $\mathrm{C}_{14} \mathrm{H}_{22} \mathrm{O}\left(\mathrm{C}_{2} \mathrm{H}_{4} \mathrm{O}\right)_{n}$, $n=9-10$, Sigma-Aldrich, USA), sodium dodecyl sulfate (SDS, $\mathrm{NaC}_{12} \mathrm{H}_{25} \mathrm{SO}_{4}$, Sigma-Aldrich, China), sodium dodecylbenzenesulfonate (SDBS, $\mathrm{C}_{18} \mathrm{H}_{29} \mathrm{NaO}_{3} \mathrm{~S}$, TCI, Japan), and 1-hexadecyl trimethyl ammonium bromide (CTAB, $\mathrm{CH}_{3}\left(\mathrm{CH}_{2}\right)_{15} \mathrm{~N}\left(\mathrm{CH}_{3}\right)_{3} \mathrm{Br}$, Alfa Aesar, Great Britain) to filtered water (obtained from a water dispenser), and the solution was dispersed using a stirrer/hot plate (PC420D, Corning, USA). Because TX100, SDS, SDBS, and CTAB produce a large amount of bubbles when they are dissolved in water, an antifoaming agent (Antifoam B Silicone Emulsion, J. T. Baker, USA) was added to suppress bubble formation and to facilitate the packaging, sampling, and testing of the solution. The antifoaming agent concentration was $15 \%$ of the weight of the added stabilizers, and the amount of the antifoaming agent was determined by the trial and error method. The aqueous solution of the stabilizer was then further diluted with filtered water to yield solutions with concentrations of $0.05,0.1,0.2,0.4$, and $0.8 \mathrm{wt} . \%$, which were used as base liquids for preparing MWNFs.

Next, a base liquid with the required weight of MWCNTs was prepared by adding MWCNTs in several installments, which effectively reduced agglomeration by reducing the probability of MWCNTs between each other combining. A stirrer/hot plate (PC420D, Corning, USA) operating at $600 \mathrm{rpm}$ with ultrasonic bath $(400 \mathrm{~W}, \mathrm{D} 400 \mathrm{H}$, TOHAMA, Taiwan) was used to disperse MWCNTs uniformly before more MWCNTs were added to the base liquid. After the concentration of MWCNTs was added to reach $0.5 \mathrm{wt} . \%$ and after 
using a homogenizer (YOM300D, Yotec, Taiwan) operating at $6000 \mathrm{rpm}$ for $30 \mathrm{~min}$, a stirrer/hot plate (PC420D, Corning, USA) operating at $600 \mathrm{rpm}$ for $1.5 \mathrm{~h}$, and an ultrasonic liquid processor (700 W, Q700, Qsonica, USA) operating for $30 \mathrm{~min}$ to ensure stable dispersion and suspension of MWCNTs in the base liquid, an intermittent oscillation process was used for the ultrasonic liquid processor (amplitude ratio: 25\%; on/off duty: 10/10 s). These dispersal devices were used three times to effectively prevent the temperature in the dispersed equipment and nanofluid from increasing, thus achieving excellent dispersion.

\section{Experimental Procedure and Design}

3.1. Experiments for Determining Basic Properties. Stabilizers affect the thermal properties and related physical and chemical properties of MWNFs $[5,6]$. First, the viscosity, density, $\mathrm{pH}$, electrical conductivity, thermal conductivity, and specific heat of aqueous solutions with different stabilizers were measured. To clarify the differences between the stabilizers, a higher stabilizer concentration (1.6 wt.\%) of the aqueous solution (six samples) was applied to reduce experimental deviation in the characteristics test of stabilizer aqueous solution. Because dyeing performance of the MWCNTs is so high that the water with a small amount of MWCNTs suspended is opaque, it is difficult to use static position method to assess the stability of MWNFs of higher concentration. Therefore, the trial and error method was used to prepare the MWNFs with different concentrations of MWCNTs without stabilizers and we waited for 24 hours and then selected the concentration showing significant precipitation as the concentration of test sample. Finally, the MWNFs with 0.5 wt.\% MWCNTs were selected as the concentration of test sample. The $\mathrm{pH}$, viscosity, and electrical conductivity of the 0.5 wt. $\%$ MWNFs with different stabilizer concentrations (30 samples) were then measured to assess the possible effects of these parameters on the stability of the MWNFs.

The thermal conductivity, density, $\mathrm{pH}$, electrical conductivity, and viscosity of the samples were measured using a thermal properties analyzer (KD2 Pro, Decagon Devices, USA; accuracy: $\pm 5.0 \%$ ), a $\mathrm{pH}$ meter (PH510, Eutech, Singapore; accuracy: $\pm 1.0 \%$ ), a liquid density meter (DA130, KEM, Japan; accuracy: $\pm 1.0 \%$ ), an electric conductivity meter (HTC-202U, HOTEC, Taiwan; accuracy: $\pm 1.0 \%$ ), and a resonant viscosity meter (Viscolite VL700HP T15-3, Hydramotion, England; accuracy: $\pm 1.0 \%)$ in an isothermal unit (P20, YSC, Taiwan), in which the sample was maintained at $25^{\circ} \mathrm{C}$ within an accuracy of $\pm 0.5^{\circ} \mathrm{C}$. To reduce measurement deviations, each parameter was measured six times. The four most concentrated measurements were then averaged and considered as the experimental value of the sample. Because the thermal properties analyzer showed high deviations, the thermal conductivity of each sample was measured 10 times for each experimental parameter, and the six most concentrated measurements were averaged as the experimental value of the sample.

The specific heat of the sample was measured using a differential scanning calorimeter (DSC, Q20, TA, USA) and a vapor compression refrigeration system (RCS40, TA, USA) in a high-purity nitrogen $(5 \mathrm{~N})$ atmosphere. The temperature accuracy and calorimetric accuracy of the DSC were $\pm 0.1^{\circ} \mathrm{C}$ and $\pm 1 \%$, respectively. The weight of samples was measured using precision electronic balance $(0.01 \mathrm{mg} / 42 \mathrm{~g}, \mathrm{GR}-202$, A\&D, Japan) and the weight of each sample was controlled at $12 \pm 1 \mathrm{mg}$. The specific heat test method is a standard reference approach, and the standard reference was pure water [30]. For obtaining the heat flow data in the temperature range of 20 to $40^{\circ} \mathrm{C}$, the experimental temperature range was $10-$ $50^{\circ} \mathrm{C}$ and the heating rate was $20^{\circ} \mathrm{C} / \mathrm{min}$. The specific heat was calculated using the heat flow data and DSC software (Universal Analysis 2000, TA, USA). To reduce measurement deviations, the specific heat experiment was conducted three times for each experimental parameter. The measured data were averaged to obtain the specific heat value of the samples.

3.2. Experiments for Determining Stability. The static position method is the most commonly used method to evaluate the stability of nanofluids. However, this method cannot evaluate the stability of nanofluids in heat exchange applications. Therefore, in this study, stability of nanofluids was determined using static and dynamic tests. First, we selected MWNFs with higher stability by using the static position method. The selected samples were then evaluated through centrifugal sedimentation, zeta potential measurement, and rheological tests to obtain the optimal stabilizer, and stabilizer concentration, and to evaluate the stability of MWNFs.

In the static position method, all samples were left standing for 30 days, and the difference in stability between samples was observed by the naked eye. However, when the difference was small, naked eye observation was difficult. Therefore, in the static position method, an ultravioletvisible-near infrared (UV/VIS/NIR) spectrometer (V670, Jasco, Japan) was used at a wavelength of $800 \mathrm{~nm}$ to measure the absorbance of each sample initially and after 1 day, 7 days, and 30 days to determine the stability of each sample. Two milliliters of each sample was filled in a standard disposable cuvette (3.0 mL, Kartell, Italy). For measuring the absorbance changes in the sample, the central location of the optical path of the spectrometer was positioned $0.5 \mathrm{~cm}$ below the liquid level of the sample.

The samples selected using the static position method were centrifuged using a digital centrifuge (EBA 20, Hettich, Germany) at a speed of $5000 \mathrm{rpm}$ for $30 \mathrm{~min}$. The amount of samples centrifuged was $10 \mathrm{~mL}$, and $0.7 \mathrm{~mL}$ of the sample was drawn using a quantitative pipette (DV-1000, HTL, Poland) from $1.0 \mathrm{~cm}$ below the level of the sample, and then to fill in a semi-micro disposable cuvette (7591-15, PlastiBrand, Germany). The UV/VIS/NIR spectrometer was used at a wavelength of $800 \mathrm{~nm}$ to measure the absorbance of samples and compare the stability of samples.

The zeta potential of the selected samples was measured using a particle size/zeta potential analyzer (SZ-100, Horiba, Japan). Because of the opacity of the MWNFs, $V_{z}$ could not be directly measured using the dynamic light scattering (DLS) method. Therefore, the test sample was centrifuged, and a clearer solution of $1.0 \mathrm{~mL}$ drawn from near the liquid surface 


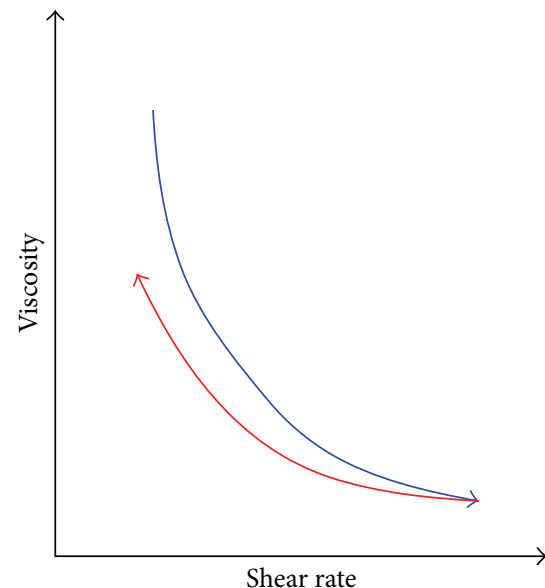

(a)

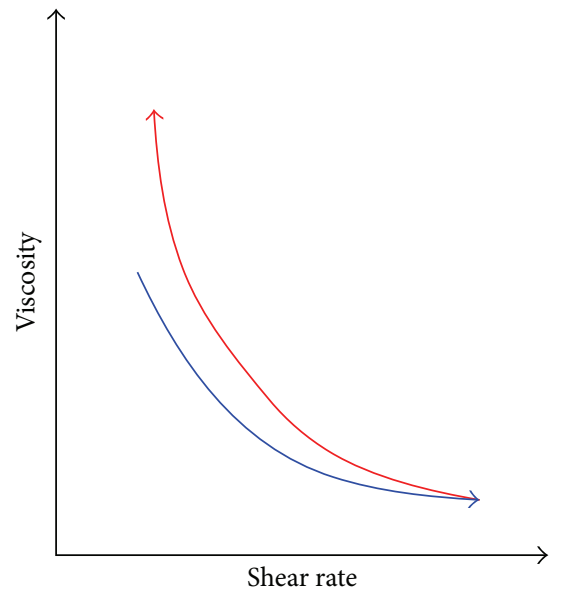

(c)

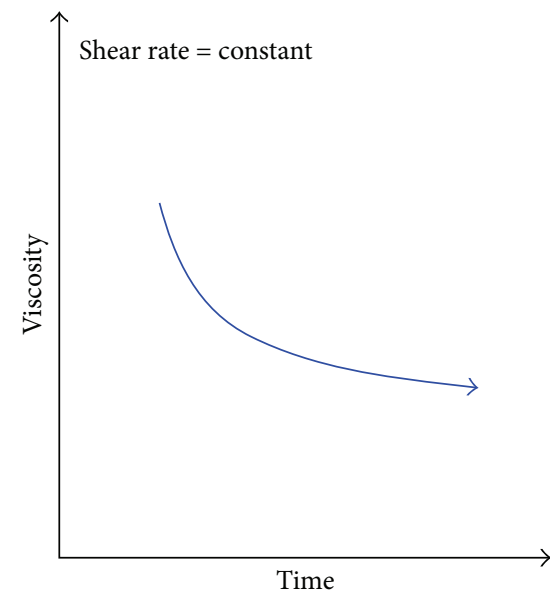

(b)

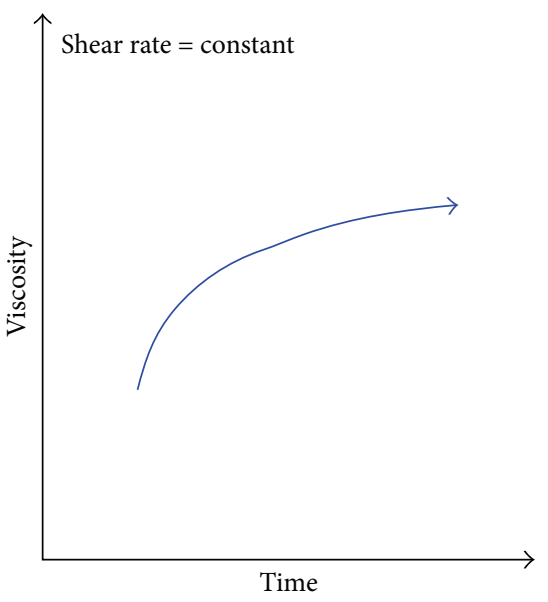

(d)

FIGURE 2: Schematic of the rheological properties of fluids: (a) thixotropic properties versus shear rate, (b) thixotropic properties versus time, (c) rheopectic properties versus shear rate, and (d) rheopectic properties versus time.

was mixed with $20 \mu \mathrm{L}$ of the original sample for use as a test sample to determine $V_{z}$. The test sample was then in an ionic state close to that of the original sample.

The rheological properties of the selected samples were determined by using a rheometer (DV3TLVCP, Brookfield, USA) with an accuracy of $\pm 1.0 \%$, and the sample temperature was controlled at $25^{\circ} \mathrm{C}$ by using an isothermal unit (HW401L, HILES, Taiwan). Structural damage and solidliquid separation in nanofluids can be evaluated by considering thixotropic or rheopectic properties of the nanofluids. In Figure 2, the thixotropic or rheopectic properties of nanofluids were tested in the following two ways. (1) Viscosity differences between nanofluids were determined according to acceleration and deceleration processes in the rheometer (shear rate dependent viscosity). (2) Viscosity differences between nanofluids at a specific shear rate were determined before and after the test period (time dependent viscosity). The smaller viscosity differences between nanofluids (low thixotropic or rheopectic properties) in the test process may indicate that the structure of the nanofluids showed little damage or that the nanofluids exhibited structural recovery, which suggested higher stability of the MWNFs.

Finally, a different test method was used to determine the stability and to select the optimal type and concentration of stabilizer. A fixed MWCNT-stabilizer concentration ratio (MWCNT: stabilizer, w/w) was determined for the different stabilizers. MWNFs with different concentrations of MWCNTs were produced with the optimal type and fixed concentration ratio of stabilizers, and their stability, thermal conductivity, and $\mathrm{pH}$ were tested to confirm the usefulness of the selected stabilizer for heat transfer applications.

3.3. Data Analysis. The UV/VIS/NIR spectrometer was used with the static positioning and centrifugal sedimentation methods to determine the difference between the absorbance of MWNFs initially $\left(\mathrm{ABS}_{i}\right)$ and at a later test time $\left(\mathrm{ABS}_{t}\right)$. The results were used to determine the stability of the MWNFs, for which (1) was used. A higher absorbance difference ratio 
TABLE 2: Characteristics of water and aqueous solutions of the stabilizers at $25^{\circ} \mathrm{C}$.

\begin{tabular}{|c|c|c|c|c|c|c|c|}
\hline \multirow{2}{*}{ Property } & \multirow{2}{*}{ Water } & \multicolumn{6}{|c|}{ Aqueous solution of stabilizers of 1.6 wt.\% } \\
\hline & & $\mathrm{CH}$ & AG & TX100 & SDS & SDBS & CTAB \\
\hline Specific heat $\left(\mathrm{kJ} / \mathrm{kg}^{\circ} \mathrm{C}\right)$ & 4.182 & 4.383 & 3.992 & 4.009 & 3.640 & 4.000 & 4.283 \\
\hline Thermal conductivity $\left(\mathrm{W} / \mathrm{m}^{\circ} \mathrm{C}\right)$ & 0.604 & 0.604 & 0.585 & 0.576 & 0.577 & 0.590 & 0.612 \\
\hline $\mathrm{pH}$ & 8.68 & 4.46 & 6.98 & 7.17 & 7.88 & 7.92 & 6.95 \\
\hline Electrical conductivity $(\mu \mathrm{S} / \mathrm{cm})$ & 92.8 & 2200 & 3020 & 105.8 & 1680 & 1970 & 1047 \\
\hline Viscosity (mPa s) & 0.8 & 1.3 & 5.9 & 1.1 & 1.1 & 1.1 & 1.2 \\
\hline Density $\left(\mathrm{kg} / \mathrm{m}^{3}\right)$ & 996.83 & 999.30 & 1003.95 & 998.03 & 998.78 & 998.95 & 996.73 \\
\hline
\end{tabular}

$\left(R_{\mathrm{ABS}}\right)$ represents a higher level of sedimentation of MWNFs after the test time and vice versa:

$$
R_{\mathrm{ABS}} \%=\left[\frac{\left(\mathrm{ABS}_{t}-\mathrm{ABS}_{i}\right)}{\mathrm{ABS}_{i}}\right] \times 100 .
$$

The rheometer was used to measure the viscosity in the acceleration and deceleration processes at different shear rates. The results were used to determine the shear rate dependence of the thixotropic or rheopectic properties, and the shear rate dependence in (2) was used for evaluating the stability of the MWNFs. A low sum of viscosity differences (DRT) represents higher stability of MWNFs and vice versa:

$$
\mathrm{DRT}=\sum\left|\mu_{\mathrm{ac}}-\mu_{\mathrm{dc}}\right|_{\text {at }} \text { the same shear rate, }
$$

where $\mu_{\mathrm{ac}}$ and $\mu_{\mathrm{dc}}$ are the accelerated viscosity and decelerated viscosity at the same shear rate, respectively.

The rheometer was used to measure the difference between viscosities at the fixed shear rate before and after the test period. The results were applied to (3) to determine the time dependence of thixotropic or rheopectic properties and to evaluate the stability of the MWNFs. A low relative standard deviation (RSD) of viscosity represents higher stability of MWNFs and vice versa:

$$
\mathrm{RSD}=\left(\frac{\mathrm{SD}}{\mu_{m}}\right) \times 100 \%,
$$

where SD is the standard deviation at a fixed shear rate for the test period and $\mu_{m}$ is the mean viscosity at the fixed shear rate during the test period.

The experimental results obtained with water were used as baseline values $\left(D_{W}\right)$ for simple comparison of the experimental data after changing the test samples $\left(D_{\text {test }}\right)$. Some of the experimental data obtained with the test samples were compared with the baseline values. The differences between the baseline and test samples are presented as the percentage $(R)$ and can be calculated as follows:

$$
R=\left[\frac{\left(D_{\text {test }}-D_{W}\right)}{D_{W}}\right] \times 100 \% .
$$

\section{Results and Discussion}

Table 2 lists the basic characteristics of water and different stabilizers in water with a concentration of $1.6 \mathrm{wt} . \%$.
The stabilizer added to the water affects the basic characteristics of the water mainly because of the characteristic of stabilizer itself, combination and chemical reaction between stabilizer and water. Adding $\mathrm{CH}$ and $\mathrm{CTAB}$ to water increased the specific heat of water: the specific heat of the aqueous solutions of $\mathrm{CH}$ and CTAB increased by more than $4.81 \%$ and $2.42 \%$, respectively. Adding $\mathrm{CH}, \mathrm{AG}, \mathrm{TX} 100, \mathrm{SDS}$, and SDBS to water reduced the thermal conductivity of water; adding $\mathrm{CTAB}$ to water led to the maximum enhancement of the thermal conductivity of water (by $1.32 \%$ ).

Stabilizers added to water reduced the $\mathrm{pH}$ of water, with the addition of $\mathrm{CH}$ leading to the maximum reduction in the $\mathrm{pH}$ of water (by $48.62 \%$ ). Adding $\mathrm{CH}$ causes substantial decrease in $\mathrm{pH}$ because the water-soluble chitosan is the chitosan (insoluble in water) treated by organic acids. Therefore, adding $\mathrm{CH}$ to the water leads to a substantial decline in $\mathrm{pH}$ of the aqueous solution.

Adding stabilizers to water increased the electrical conductivity of water, and adding AG led to the maximum enhancement of the electrical conductivity of water (by 3154.31\%). Except TX100 which is a nonionic stabilizer, the other stabilizers are anionic (SDS, SDBS, and AG) or cationic $(\mathrm{CH}$ and $\mathrm{CTAB})$ stabilizers. The electrical conductivity of the water increases with the number of ions in the water. Therefore, adding an anionic or cationic stabilizer in water substantially increases the electrical conductivity of the water.

Stabilizers are added to water to increase the viscosity of water. In this study, AG addition showed the maximum enhancement of the viscosity of water (by 637.50\%). AG is capable of absorbing 200-300 times its own weight in water to form a gum of high viscosity. Therefore, adding $\mathrm{AG}$ in the water will greatly increase the viscosity of water. Furthermore, $\mathrm{CH}$ is generated by the hydrolysis of chitosan to form carbohydrate with low degree of polymerization $(n=$ 2 20) whose viscosity is less than AG but is still higher than other stabilizers. Therefore, the viscosity of the $\mathrm{CH}$ aqueous solution is higher than $62.50 \%$ of water. Other stabilizers (TX100, SDS, SDBS, and CTAB) that belong to the surfactant (the surfactant is dissolved in water and has a lower increase in the viscosity) are used in the detergent and buffer solution or the like purposes.

The effect of stabilizers on water density is unclear. This phenomenon is mainly due to minor differences of density between the stabilizer and water, and moreover, the added amount of stabilizers is small. However, AG addition led 
TABLE 3: Characteristics of MWNFs containing different types and concentrations of stabilizer at $25^{\circ} \mathrm{C}$.

\begin{tabular}{|c|c|c|c|c|c|c|}
\hline \multirow{2}{*}{ Property } & \multirow{2}{*}{ Stabilizer } & \multicolumn{5}{|c|}{ Stabilizer concentration (wt.\%) } \\
\hline & & 0.05 & 0.1 & 0.2 & 0.4 & 0.8 \\
\hline \multirow{6}{*}{ Viscosity (mPa s) } & $\mathrm{CH}$ & 1.2 & 1 & 0.9 & 1 & 1.1 \\
\hline & AG & 1.2 & 1.2 & 1.4 & 1.9 & 3.0 \\
\hline & TX100 & 0.9 & 0.9 & 0.9 & 0.9 & 1.0 \\
\hline & SDS & 0.9 & 0.9 & 0.9 & 0.9 & 1.0 \\
\hline & SDBS & 0.9 & 0.9 & 0.9 & 0.9 & 1.0 \\
\hline & CTAB & 0.9 & 0.9 & 0.9 & 0.9 & 0.9 \\
\hline \multirow{6}{*}{$\mathrm{pH}$} & $\mathrm{CH}$ & 6.51 & 4.52 & 4.32 & 4.24 & 4.22 \\
\hline & AG & 7.87 & 7.83 & 7.76 & 7.75 & 7.69 \\
\hline & TX100 & 7.62 & 7.63 & 7.64 & 7.32 & 7.12 \\
\hline & SDS & 7.32 & 7.34 & 7.41 & 7.74 & 7.83 \\
\hline & SDBS & 7.62 & 7.71 & 7.72 & 7.72 & 7.91 \\
\hline & CATB & 7.32 & 7.42 & 7.44 & 7.48 & 7.52 \\
\hline \multirow{6}{*}{ Electrical conductivity $(\mu \mathrm{S} / \mathrm{cm})$} & $\mathrm{CH}$ & 225 & 309 & 511 & 820 & 1410 \\
\hline & AG & 390 & 539 & 803 & 1238 & 1970 \\
\hline & TX100 & 201 & 196 & 134 & 125 & 126 \\
\hline & SDS & 343 & 430 & 567 & 885 & 1130 \\
\hline & SDBS & 330 & 357 & 402 & 610 & 1120 \\
\hline & CATB & 196 & 216 & 263 & 375 & 608 \\
\hline
\end{tabular}

to the maximum enhancement of the density of water (by $0.71 \%)$.

High viscosity results in a pressure drop in the pipeline in the heat exchange system; therefore, AG is not suitable for producing MWNFs that can be used as heat exchange working fluids. Furthermore, adding $\mathrm{CH}$ to water significantly lowers the $\mathrm{pH}$ of water; therefore, when $\mathrm{CH}$ is used to produce MWNFs that can be used as heat exchange working fluids, the material of the heat exchange system must be chosen carefully to avoid corrosion problems.

Table 3 lists the basic characteristics of MWNFs with 0.5 wt\% MWCNTs; the MWNFs contain different stabilizers and stabilizer concentrations. The viscosity of MWNFs containing AG was considerably higher than that of water, whereas the $\mathrm{pH}$ of MWNFs containing $\mathrm{CH}$ was much lower than that of water. The viscosity of MWNFs with 0.05 and 0.1 wt. \% $\mathrm{CH}$ is higher than that of MWNFs with $0.2 \mathrm{wt} . \% \mathrm{CH}$ mainly because of poor dispersion performance. Aggregation of MWCNTs in MWNFs will cause viscosity of MWNFs to increase. The viscosity and $\mathrm{pH}$ of most of the other samples were within the acceptable range. However, the electrical conductivity of MWNFs containing stabilizers was considerably higher than that of water. The addition of AG and $\mathrm{CH}$ will significantly increase the viscosity and reduce $\mathrm{pH}$ of MWNFs, respectively; the trends are the same as those in Table 2. MWNFs belong to the solid-liquid mixture whose viscosity is usually higher than the base liquid. In addition, because the use of chemical method to prepare MWCNTs typically adds acids, the $\mathrm{pH}$ of MWNFs is still affected by small amount of residue even if MWCNTs are cleaned tending to neutral. Furthermore, because MWCNTs have high electrical conductivity, MWCNTs added to the base liquid will increase the electrical conductivity of the base liquid. The MWNFs should have near-neutral $\mathrm{pH}$ and not too much viscosity increase ratio to reduce the pipeline corrosion and higher pumping power when the MWNFs will be applied to the heat exchange system.

Figure 3 shows a photograph of the static position experiment after 30 days; MWNFs with different stabilizers and concentrations were used in the experiment. Samples with AG, TX100, and CTAB exhibited sedimentation. Figures 4, 5, and 6 show the absorbance difference ratio (equation (1)) of MWNFs in the static position experiment for 1 day, 7 days, and 30 days, respectively. The suspension performance of MWCNTs with stabilizers in MWNFs is mainly determined by electrostatic and interface characteristics between MWCNTs and stabilizers. The repulsive force between MWCNTs coated with stabilizers is higher than attractive force which will be able to effectively prevent the agglomeration of MWCNTs and thus reduce the sedimentation of MWCNTs. However, MWCNTs with different types and concentrations of stabilizers have different electrostatic and interface characteristics, and therefore the different types and concentrations of stabilizers affect suspension performance of MWCNTs in MWNFs. With an increase in the standing time, the suspension performance of each MWNF showed gradual degradation. MWNFs containing $\mathrm{CH}$, SDS, and SDBS demonstrated higher overall stability. Therefore, subsequent stability test was conducted only for these MWNFs.

Figure 7 displays the absorbance difference ratio of MWNFs containing CH, SDS, and SDBS before and after centrifugation. The stability did not significantly change for stabilizer concentrations greater than $0.2 \mathrm{wt} \%$. The MWNFs with excellent stability can represent the excellent combination between on the surface of MWCNTs and base liquid that 
$\mathrm{CH}$

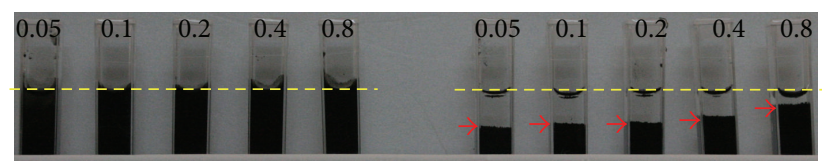

(a)

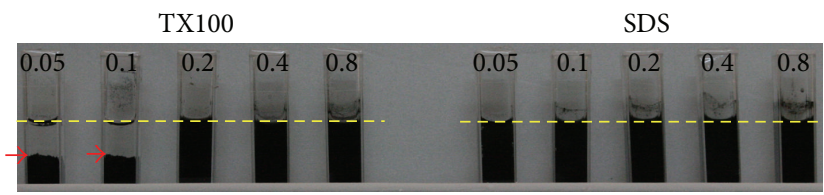

(b)

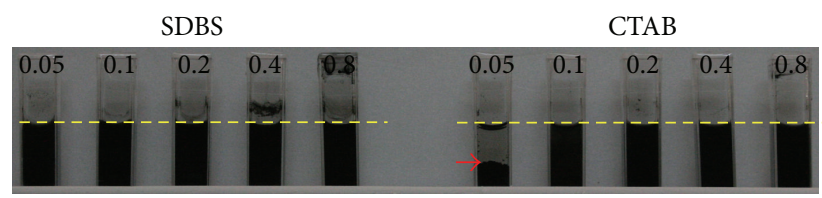

(c)

FIGURE 3: Photograph of experimental results of the static position method after 30 days.

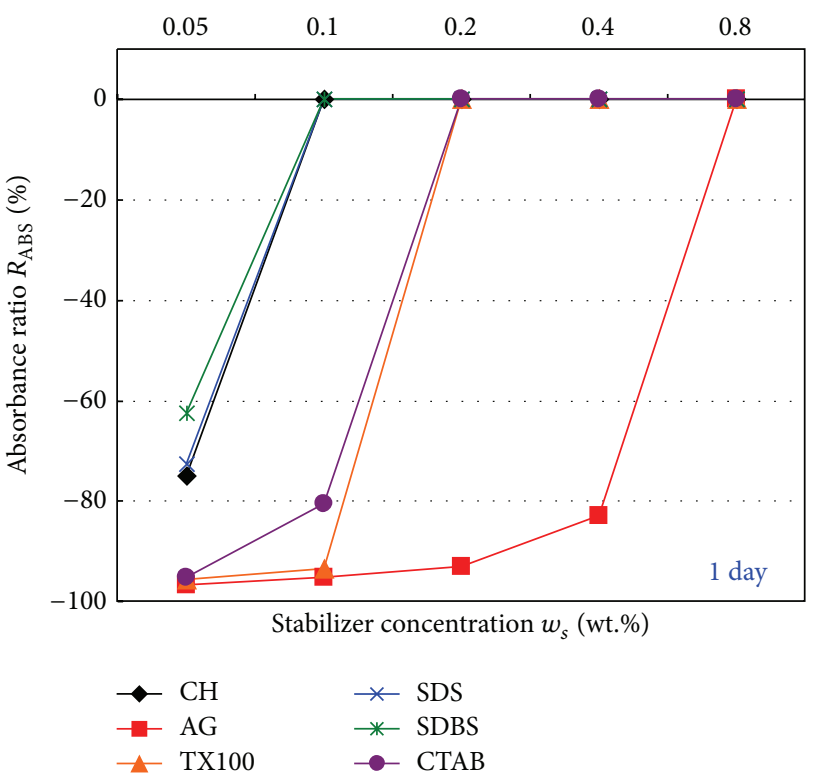

Figure 4: Absorbance difference ratio among MWNFs in the static position experiment for different types and concentrations of stabilizers after 1 day.

can resist solid-liquid separation of MWNFs when MWNFs in the flow state or exerted by force. However, MWCNTs with different types and concentrations of stabilizers have different combinations, and therefore the different types and concentrations of stabilizers affect stability of the solid-liquid structure for MWNFs.

Figure 8 illustrates the zeta potential $\left(V_{z}\right)$ of MWNFs containing $\mathrm{CH}, \mathrm{SDS}$, and SDBS. $\mathrm{CH}$ is a cationic stabilizer; therefore, it has a positive $V_{z}$; SDS and SDBS are anionic stabilizers; thus, they yield a negative $V_{z}$. The addition of

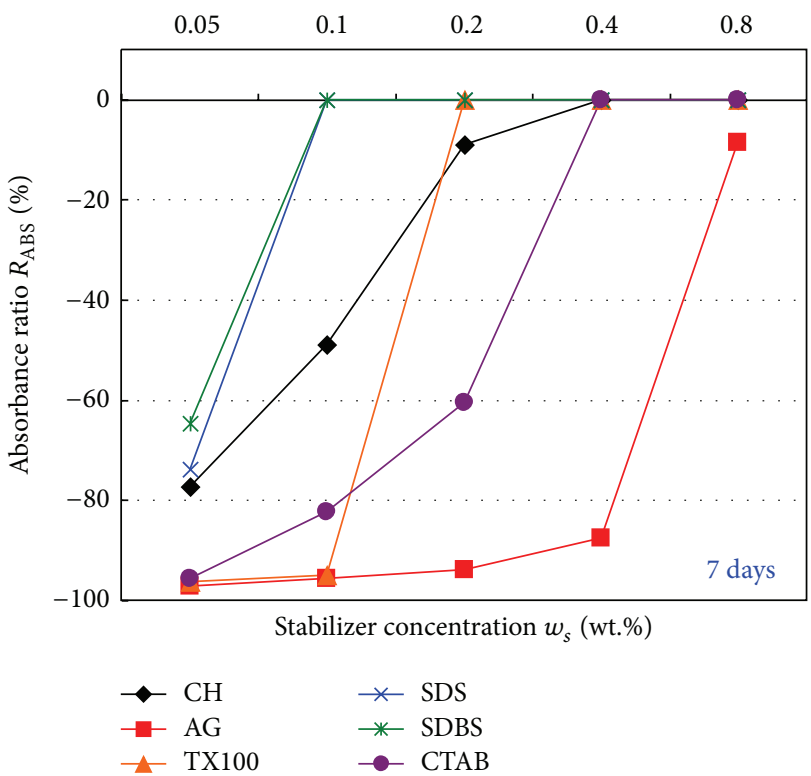

FIGURE 5: Absorbance difference ratio among MWNFs in the static position experiment for different types and concentrations of stabilizers after 7 days.

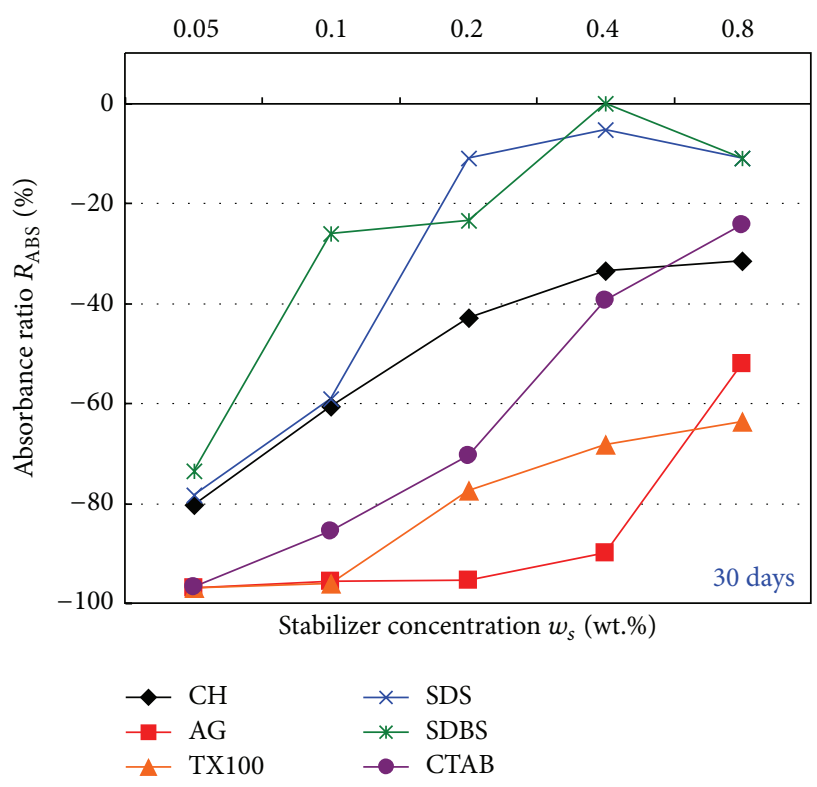

Figure 6: Absorbance difference ratio among MWNFs in the static position experiment for different types and concentrations of stabilizers after 30 days.

$\mathrm{CH}, \mathrm{SDS}$, and SDBS with concentrations of $0.2,0.8$, and $0.2 \mathrm{wt} \%$ yielded MWNFs showing the maximum $V_{z}$ values; the maximum $V_{z}$ values were $46.2,-85.6$, and $-80.0 \mathrm{mV}$, respectively. The $V_{z}$ value of nanofluids is within the range from \pm 40 to $\pm 60 \mathrm{mV}$, denoting that nanofluids demonstrate favorable stability. When $V_{z}$ of nanofluids is greater than $\pm 60 \mathrm{mV}$, the nanofluids exhibit excellent stability [25, 26]. The experimentally obtained $V_{z}$ values indicated that MWNFs 


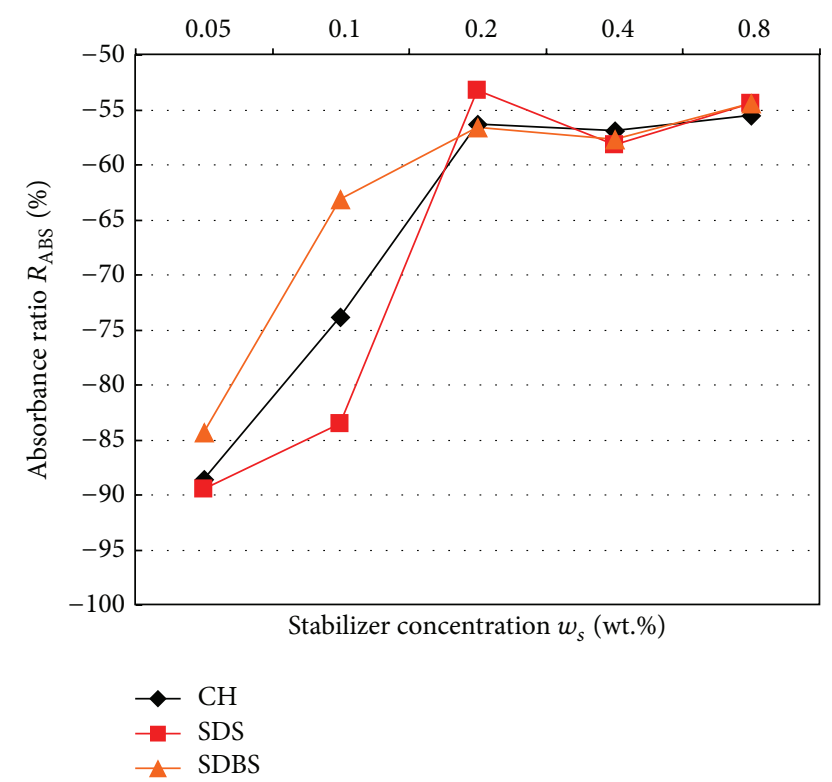

FIGURE 7: Absorbance difference ratio among the MWNFs for the centrifugal sedimentation method for different types and concentrations of stabilizers.

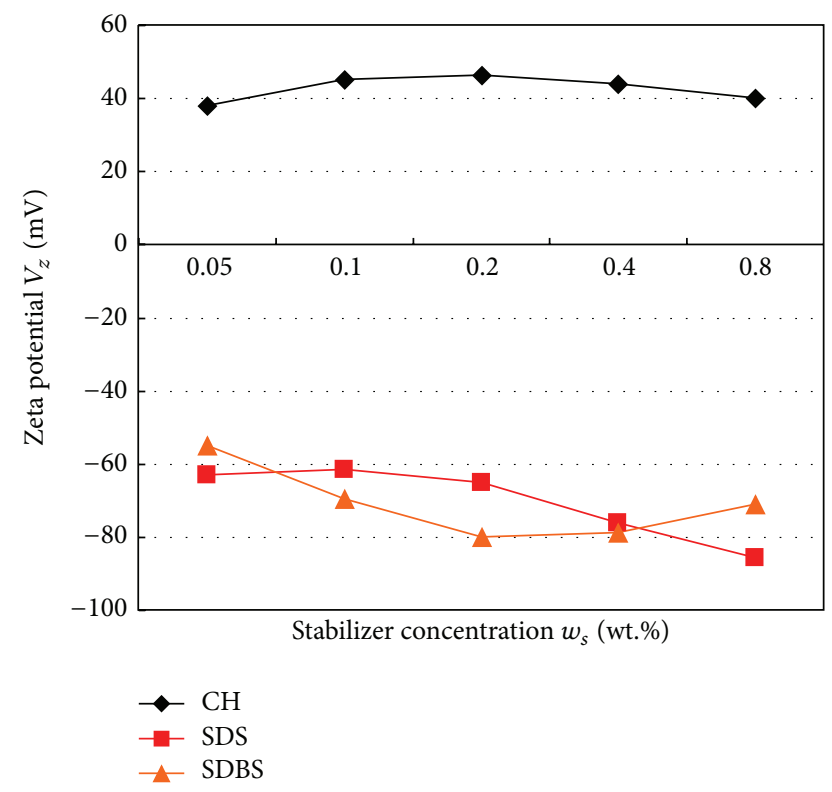

FIgURE 8: Zeta potential of the MWNFs for different types and concentrations of stabilizers.

containing SDS and SDBS showed higher stability compared with MWNFs containing $\mathrm{CH}$.

Figures 9, 10, and 11 illustrate the viscosity changes in MWNFs with different concentrations of $\mathrm{CH}$, SDS, and SDBS for different shear rates. The MWNFs with $\mathrm{CH}$ concentrations of 0.05 and $0.1 \mathrm{wt} \%$ exhibited rheopectic properties (the decelerated viscosity is greater than the accelerated viscosity), and the change ratio of the viscosity for MWNFs with a stabilizer concentration of $0.1 \mathrm{wt} \%$ was at the minimum. MWNFs containing SDS showed thixotropic properties, and

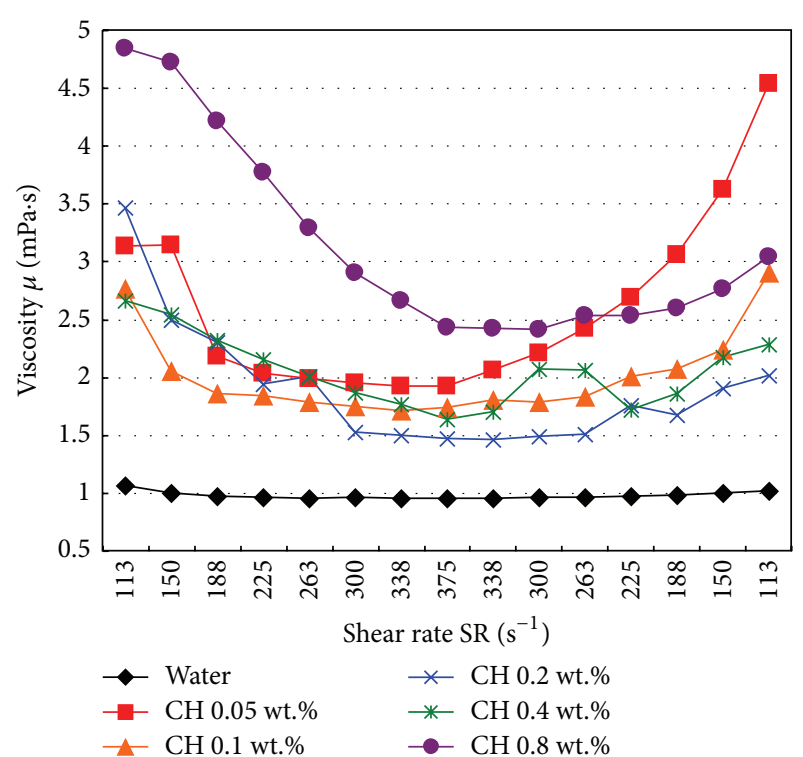

FIGURE 9: Viscosity of MWNFs containing $\mathrm{CH}$ at different shear rates.

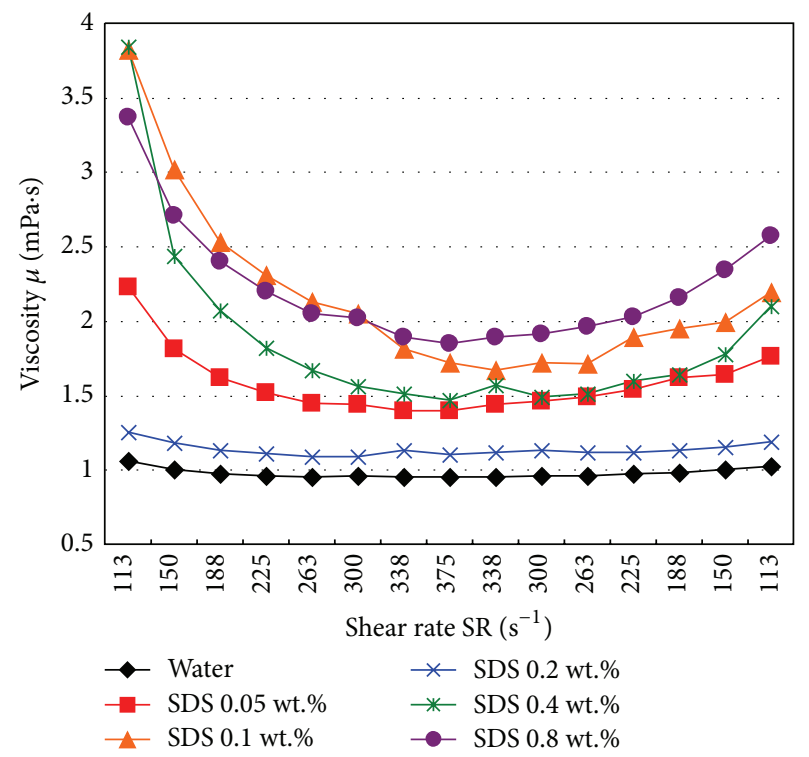

FIGURE 10: Viscosity of MWNFs containing SDS at different shear rates.

the change and increase ratios of the viscosity for MWNFs with $0.2 \mathrm{wt} \%$ SDS were at the minimum. MWNFs containing SDBS exhibited thixotropic properties, and the change and increase ratios of the viscosity of MWNFs containing $0.4 \mathrm{wt} \%$ SDS were at the minimum. The smaller viscosity differences between nanofluids (low thixotropic or rheopectic properties) in the test process indicate that the structure of the MWNFs showed little damage or that the nanofluids exhibited structural recovery, which suggested higher stability of the MWNFs. Therefore, the optimal concentrations of $\mathrm{CH}$, SDS, and SDBS for the MWNFs were 0.1, 0.2, and $0.4 \mathrm{wt} \%$, respectively. 


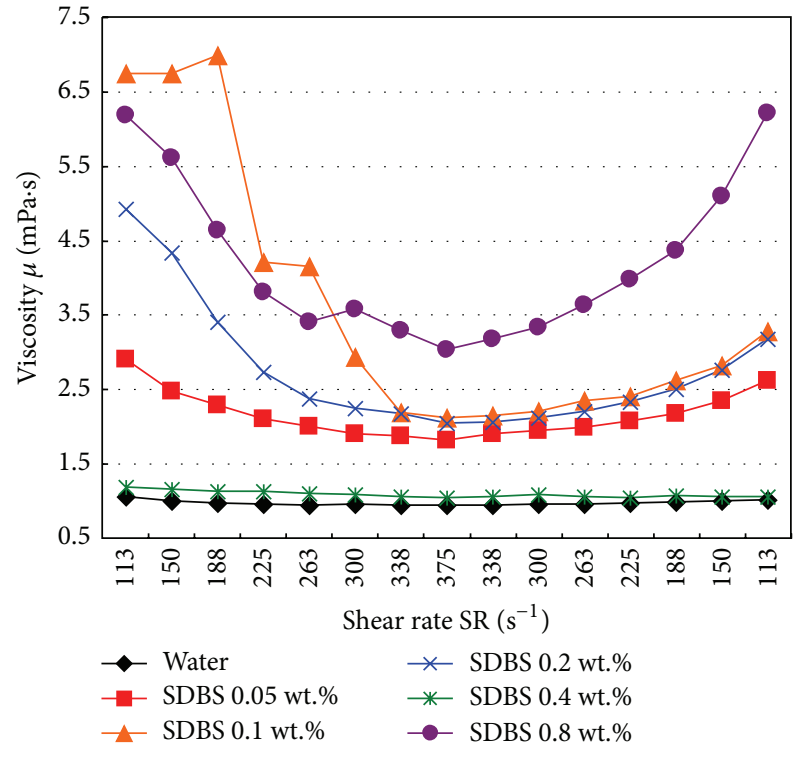

FIGURE 11: Viscosity of MWNFs containing SDBS at different shear rates.

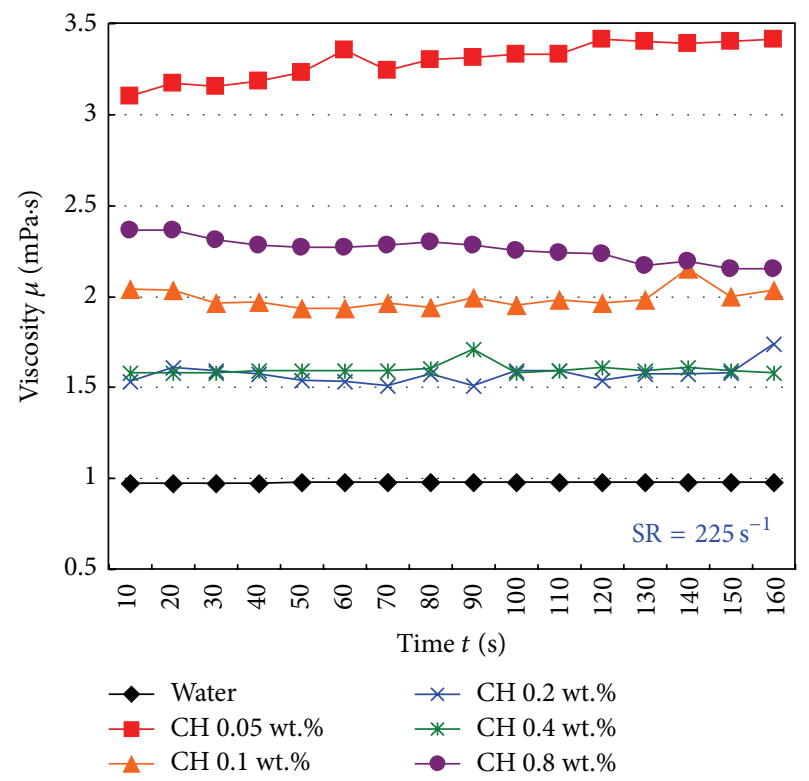

FIGURE 12: Viscosity of MWNFs containing $\mathrm{CH}$ at a specific shear rate during the test period.

Figures 12,13 , and 14 illustrate the viscosity change in MWNFs for different concentrations of $\mathrm{CH}$, SDS, and SDBS for a specific shear rate $\left(225 \mathrm{~s}^{-1}\right)$. The change and increase ratios of the viscosity of MWNFs with 0.2 and $0.4 \mathrm{wt} \%$ $\mathrm{CH}$ were at the minimum. The change and increase ratios of the viscosity of MWNFs containing $0.2 \mathrm{wt} \%$ SDS were at the minimum. The change and increase ratios of the viscosity of MWNFs containing $0.4 \mathrm{wt} \%$ SDS were at the minimum. Therefore, the optimal concentrations of $\mathrm{CH}$, SDS, and SDBS for the MWNFs were 0.2 and $0.4,0.2$, and $0.4 \mathrm{wt} \%$, respectively.

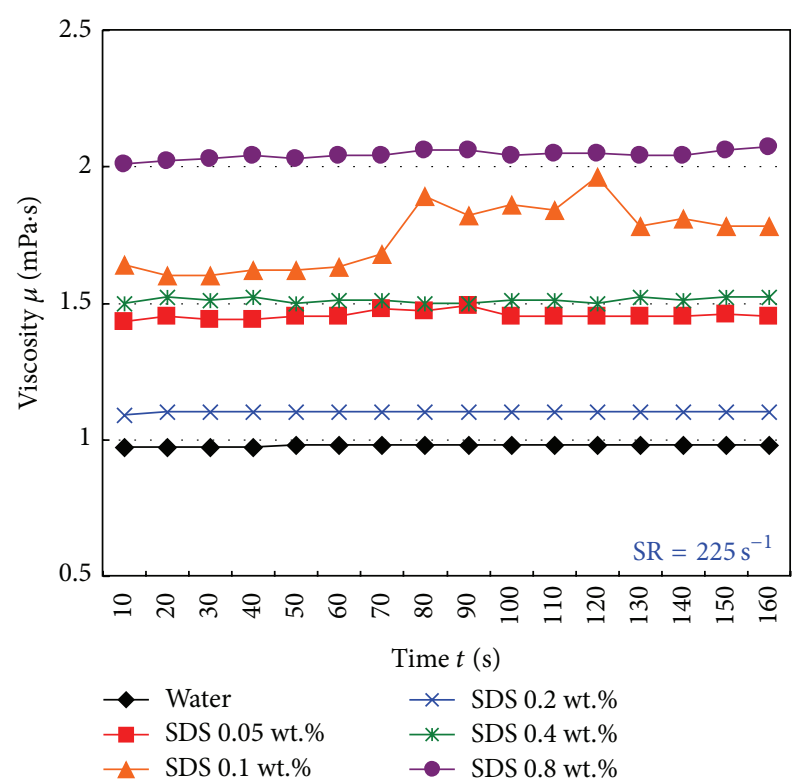

FIGURE 13: Viscosity of MWNFs containing SDS at a specific shear rate during the test period.

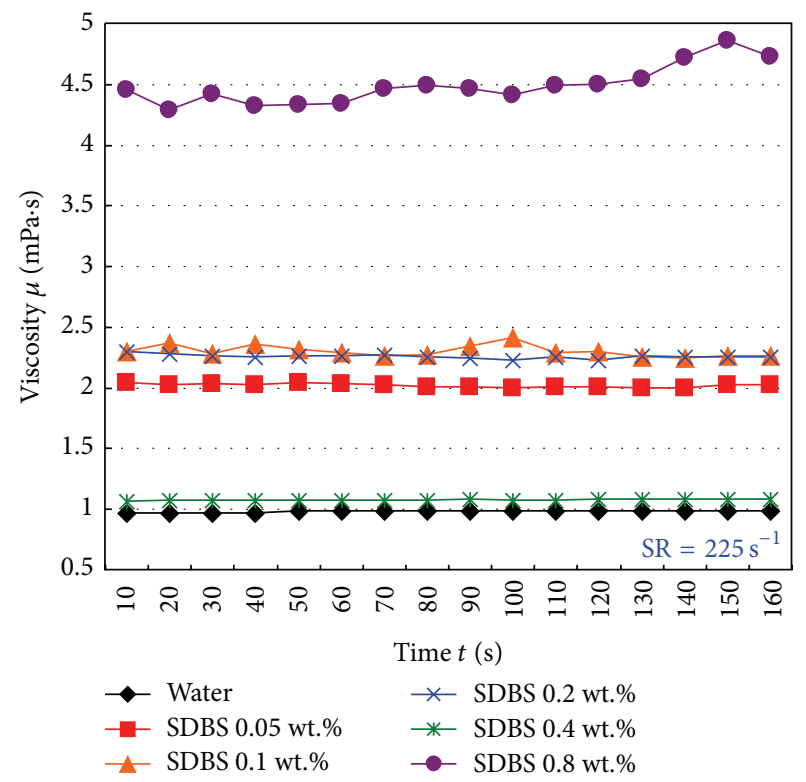

FIGURE 14: Viscosity of MWNFs containing SDBS at a specific shear rate during the test period.

Table 4 lists the experimental results for the stability of 0.5 wt. \% MWNFs containing CH, SDS, and SDBS. For determining the optimal concentration of the stabilizer, a sortweighted approach was used, and five test results (marked in bold font) for the stability were considered simultaneously. The results showed that the optimal concentrations of $\mathrm{CH}$, SDS, and SDBS were $0.4,0.2$, and $0.4 \mathrm{wt} \%$, respectively. Furthermore, adding $\mathrm{CH}$ with the optimal concentration increased the viscosity by $63.43 \%$ compared with that of water; therefore, $\mathrm{CH}$ will not be used in future experiments because of the extremely high enhancement of the viscosity 
TABLE 4: Results for the stability of MWNFs.

\begin{tabular}{|c|c|c|c|c|c|c|}
\hline \multirow{2}{*}{ Stabilizer } & \multirow{2}{*}{ Parameters } & \multicolumn{5}{|c|}{ Stabilizer concentration (wt.\%) } \\
\hline & & 0.05 & 0.1 & 0.2 & 0.4 & 0.8 \\
\hline \multirow{7}{*}{$\mathrm{CH}$} & $\mathbf{R}_{\mathrm{ABS}}(\%)^{*}$ & -80.27 & -60.71 & -42.85 & -33.44 & -31.56 \\
\hline & $\mathbf{R}_{\mathrm{ABS}}(\%)^{* *}$ & -88.60 & -73.91 & -56.29 & -56.92 & -55.57 \\
\hline & $\mathbf{V}_{\mathrm{z}}(\mathbf{m V})$ & 38.0 & 45.1 & 46.2 & 44.0 & 40.0 \\
\hline & DRT & 4.26 & 0.88 & 3.44 & 1.96 & 8.10 \\
\hline & $\mu_{m}(\mathrm{mPas})$ & 3.29 & 1.99 & 1.57 & 1.60 & 2.26 \\
\hline & $R_{\mu_{m}}(\%)$ & 236.96 & 103.32 & 60.74 & 63.43 & 130.75 \\
\hline & RSD (\%) & 3.12 & 2.78 & 3.45 & 1.97 & 2.89 \\
\hline \multirow{7}{*}{ SDS } & $\mathbf{R}_{\mathrm{ABS}}(\%)^{*}$ & -78.34 & -59.09 & -11.04 & -5.40 & -10.99 \\
\hline & $\mathbf{R}_{\mathrm{ABS}}(\%)^{* *}$ & -89.46 & -83.59 & -53.31 & -58.17 & -54.44 \\
\hline & $\mathrm{V}_{\mathrm{z}}(\mathrm{mV})$ & -63.0 & -61.7 & -65.0 & -76.3 & -85.6 \\
\hline & DRT & 0.76 & 4.55 & 0.18 & 3.34 & 1.78 \\
\hline & $\mu_{m}(\mathrm{mPa} \cdot \mathrm{s})$ & 1.45 & 1.74 & 1.10 & 1.51 & 2.04 \\
\hline & $R_{\mu_{m}}(\%)$ & 48.72 & 78.45 & 12.47 & 54.48 & 108.95 \\
\hline & RSD (\%) & 1.03 & 6.70 & 0.23 & 0.54 & 0.77 \\
\hline \multirow{7}{*}{ SDBS } & $\mathbf{R}_{\mathrm{ABS}}(\%)^{*}$ & -73.60 & -25.98 & -23.41 & 0 & -10.98 \\
\hline & $\mathbf{R}_{\mathrm{ABS}}(\%)^{* *}$ & -84.32 & -63.15 & -56.62 & -57.71 & -54.44 \\
\hline & $\mathbf{V}_{\mathrm{z}}(\mathbf{m V})$ & -54.9 & -69.5 & -80.0 & -78.9 & -71.0 \\
\hline & DRT & 0.65 & 16.15 & 5.05 & 0.42 & 1.55 \\
\hline & $\mu_{m}(\mathrm{mPas})$ & 2.02 & 2.30 & 2.26 & 1.07 & 4.49 \\
\hline & $R_{\mu_{m}}(\%)$ & 106.39 & 135.23 & 130.75 & 9.78 & 359.14 \\
\hline & RSD (\%) & 0.64 & 2.09 & 0.78 & 0.56 & 3.55 \\
\hline \multirow{3}{*}{ Water } & DRT & & & 0.07 & & \\
\hline & $\mu_{m}(\mathrm{mPas})$ & & & 0.98 & & \\
\hline & RSD (\%) & & & 0.46 & & \\
\hline
\end{tabular}

$R_{\mathrm{ABS}}(\%)^{*}$ is absorbance ratio of static position method after 30 days.

$R_{\mathrm{ABS}}(\%)^{* *}$ is absorbance ratio after centrifugation at $5000 \mathrm{rpm}$ and 30 minutes.

Viscosity $\left(\mu_{m}\right)$ is mean viscosity at shear rate of $225 \mathrm{~s}^{-1}$ in 160 seconds.

$\left(R_{\mu_{m}}\right)$, which may result in a considerable pressure drop in heat transfer applications.

Generally, the concentration of the added stabilizer should increase with the MWCNTs concentration, and it is possible to fix the MWCNTs-stabilizer concentration ratio at a specific value. The optimal ratios of the MWCNTs concentration to the stabilizer concentration for SDS and SDBS were 5:2 (0.5 wt.\% MWCNTs:0.2 wt.\% SDS) and 5 : 4 (0.5 wt.\% MWCNTs : 0.4 wt.\% SDBS), respectively, which showed excellent stability when they were evaluated by static position, centrifugal sedimentation, zeta potential, and rheological experiments at the same time. The optimal concentration ratios of MWCNTs-SDS and MWCNTs-SDBS were used to prepare MWNFs with different MWCNTs concentrations $(0.25,0.5$, and $1.0 \mathrm{wt} . \%)$, and these MWNFs were used to conduct subsequent experiments pertaining to static positioning, thermal conductivity, and $\mathrm{pH}$ to identify the optimal stabilizer and confirm that the use of the optimal concentration ratio leads to MWNFs with excellent and stable performance.

Figure 15 shows the absorbance difference ratio of MWNFs containing SDS and SDBS in the static position

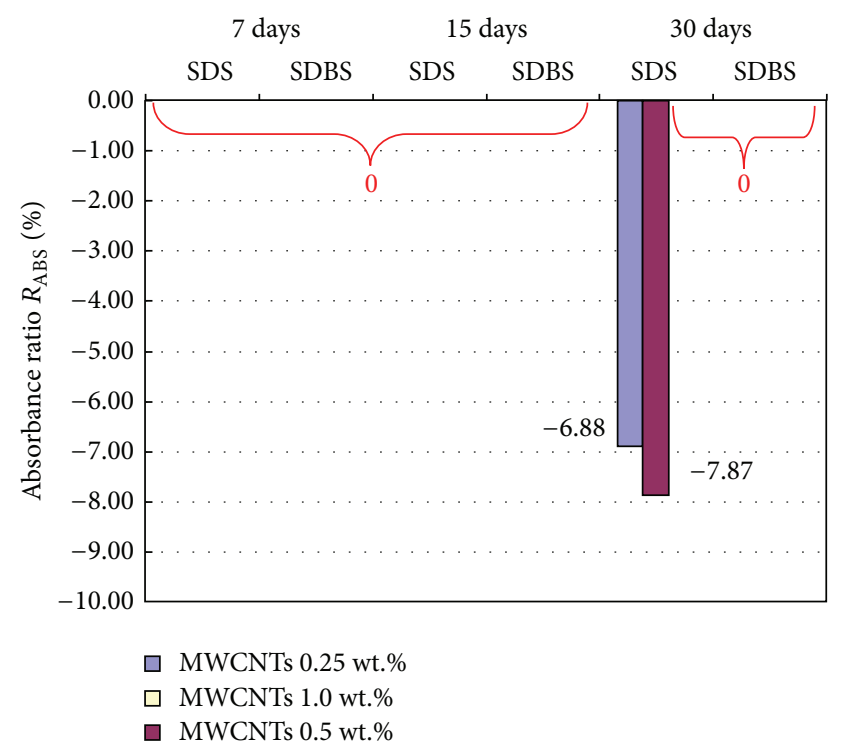

FIgURE 15: Absorbance difference ratios among the MWNFs containing SDS and SDBS in the static position experiment for different MWCNTs concentrations. 


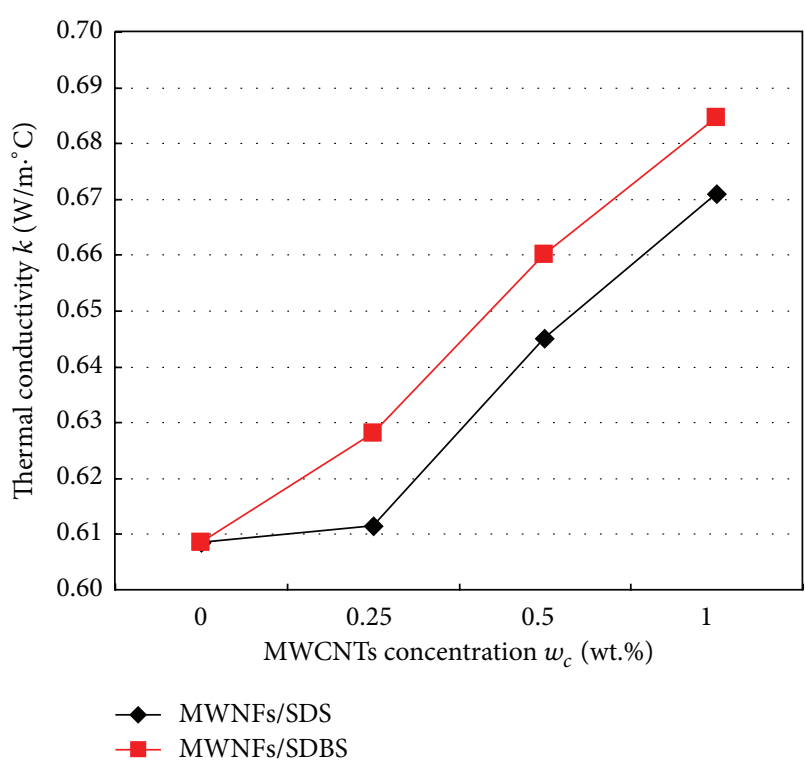

FIGURE 16: Thermal conductivity of the MWNFs containing SDS and SDBS at different MWCNTs concentrations at $25^{\circ} \mathrm{C}$.

experiment for 7, 15 and 30 days. The difference in the stability after 7 and 15 days between MWNFs containing SDS and SDBS was the same. The stability of MWNFs with 0.25 and 0.5 wt.\% MWCNTs containing SDBS was higher than that of MWNFs with 0.25 and 0.5 wt.\% MWCNTs containing SDS after 30 days. The results of the static position experiment confirmed that it was feasible to use a fixed optimal concentration ratio to prepare MWNFs with excellent stability.

Figure 16 illustrates the thermal conductivity of MWNFs containing SDS and SDBS for different MWCNT concentrations at $25^{\circ} \mathrm{C}$. MWNFs with higher MWCNTs concentrations exhibited higher thermal conductivity. Thermal conductivity of nanofluids using the same nanoadditives is mainly dependent on the concentration of nanoadditives, dispersion, suspension performance, sample temperature, and solidliquid interface characteristics (i.e., the interface layer thickness, thermal properties, and combination). MWNFs with MWCNTs concentrations of $0.25,0.5$, and $1.0 \mathrm{wt} \%$ were prepared using an aqueous SDS solution with an MWCNTsSDS concentration ratio of $5: 2$; the thermal conductivity values of the MWNFs were greater than that of water at $25^{\circ} \mathrm{C}$ by $0.49 \%, 6.00 \%$, and $10.27 \%$, respectively. MWNFs with MWCNTs concentrations of $0.25,0.5$, and $1.0 \mathrm{wt} \%$ were prepared using an aqueous SDBS solution with an MWCNTs-SDBS concentration ratio of $5: 4$; the thermal conductivity values of the MWNFs were greater than that of water at $25^{\circ} \mathrm{C}$ by $3.20 \%, 8.46 \%$, and $12.49 \%$, respectively. The maximum thermal conductivity of MWNFs containing SDBS was higher than that of MWNFs containing SDS by $2.01 \%$.

Figure 17 depicts the $\mathrm{pH}$ of MWNFs containing SDS and SDBS for different MWCNT concentrations at $25^{\circ} \mathrm{C}$. The difference in $\mathrm{pH}$ was not significant at different MWCNT concentrations because the MWCNT-stabilizer concentration ratio was constant. Overall, the $\mathrm{pH}$ of MWNFs containing SDBS was slightly higher than that of MWNFs containing

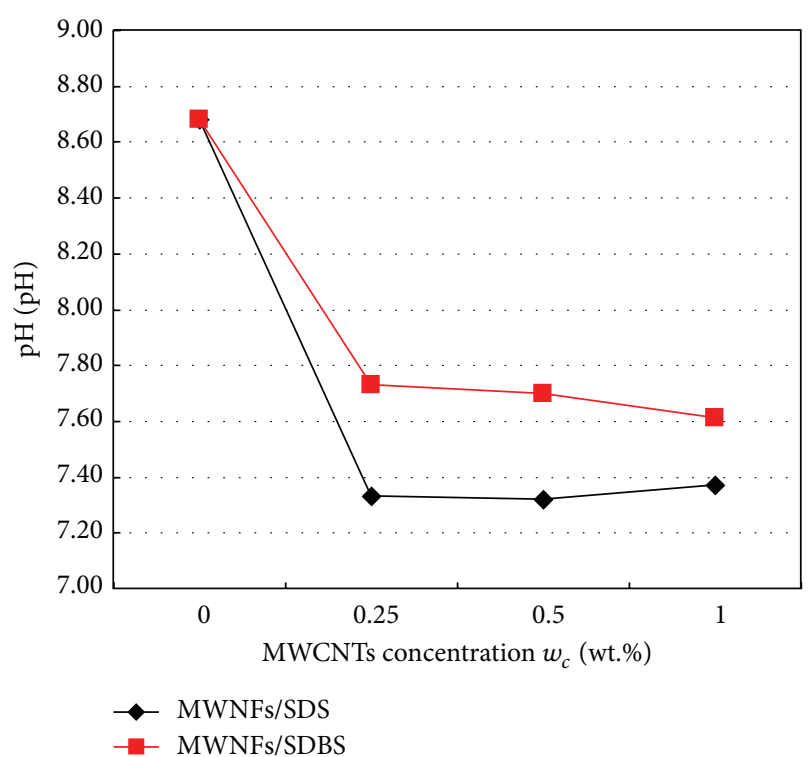

FIGURE 17: Values of $\mathrm{pH}$ of the MWNFs containing SDS and SDBS at different MWCNTs concentrations at $25^{\circ} \mathrm{C}$.

SDS; the maximum $\mathrm{pH}$ of MWNFs containing SDBS was higher by $5.17 \%$. The $\mathrm{pH}$ of MWNFs containing SDS and SDBS was near the neutral range ( $\mathrm{pH} 7.32-7.73)$; therefore, using these MWNFs should not cause pipeline corrosion problems. There are minor differences in the stability and $\mathrm{pH}$ between MWNFs containing SDS and those containing SDBS. In particular, SDBS is identified as the optimal stabilizer for preparing MWNFs because the thermal conductivity of MWNFs containing SDBS is higher than that of MWNFs containing SDS.

\section{Conclusion}

In this study, we used a two-step synthetic process to prepare MWNFs by using different stabilizers. The characteristics of aqueous stabilizer solutions and MWNFs were examined using suitable instruments and test methods. The findings of this study are summarized as follows.

(1) The viscosity, electrical conductivity, and $\mathrm{pH}$ of MWNFs are considerably different when different stabilizers are used. The maximum increase ratios of the viscosity and electrical conductivity for MWNFs were observed for $A G$, and the maximum decrease ratio of the $\mathrm{pH}$ for MWNFs was determined for $\mathrm{CH}$.

(2) Based on the static position experiment, MWNFs with $\mathrm{CH}, \mathrm{SDS}$, and SDBS demonstrate higher stability.

(3) The static and dynamic tests showed that MWNFs containing SDS and SDBS demonstrate optimal stability.

(4) The optimal MWCNT-stabilizer ratios for MWNFs containing SDS and SDBS were $5: 2$ and $5: 4$ by weight, respectively. 
(5) The static position experiment confirmed that using a fixed optimal concentration ratio is feasible for preparing MWNFs with excellent stability.

(6) The thermal conductivity values of MWNFs containing different stabilizers indicated that the most suitable stabilizer for MWNFs was SDBS. The maximum thermal conductivity of MWNFs containing SDBS was higher than the thermal conductivity of water at $25^{\circ} \mathrm{C}$ by $12.49 \%$.

(7) The $\mathrm{pH}$ of MWNFs containing SDS and SDBS was near the neutral range; therefore, using these MWNFs should not cause pipeline corrosion problems.

\section{Conflict of Interests}

The authors declare that there is no conflict of interests regarding the publication of this paper.

\section{Acknowledgment}

The authors would like to thank the Ministry of Science and Technology of Taiwan for their financial support to this research under Contract no. NSC 101-2221-E-003-011-MY2.

\section{References}

[1] J. M. Wu and J. Zhao, "A review of nanofluid heat transfer and critical heat flux enhancement-research gap to engineering application," Progress in Nuclear Energy, vol. 66, pp. 13-24, 2013.

[2] O. Mahian, A. Kianifar, C. Kleinstreuer et al., "A review of entropy generation in nanofluid flow," International Journal of Heat and Mass Transfer, vol. 65, pp. 514-532, 2013.

[3] A. M. Hussein, K. V. Sharma, R. A. Bakar, and K. Kadirgama, "A review of forced convection heat transfer enhancement and hydrodynamic characteristics of a nanofluid," Renewable and Sustainable Energy Reviews, vol. 29, pp. 734-743, 2014.

[4] Z. Haddad, C. Abid, H. F. Oztop, and A. Mataoui, "A review on how the researchers prepare their nanofluids," International Journal of Thermal Sciences, vol. 76, pp. 168-189, 2014.

[5] W. Duangthongsuk and S. Wongwises, "Heat transfer enhancement and pressure drop characteristics of $\mathrm{TiO}_{2}$-water nanofluid in a double-tube counter flow heat exchanger," International Journal of Heat and Mass Transfer, vol. 52, no. 7-8, pp. 20592067, 2009.

[6] R. Lotfi, A. M. Rashidi, and A. Amrollahi, "Experimental study on the heat transfer enhancement of MWNT-water nanofluid in a shell and tube heat exchanger," International Communications in Heat and Mass Transfer, vol. 39, no. 1, pp. 108-111, 2012.

[7] D. Zhu, X. Li, N. Wang, X. Wang, J. Gao, and H. Li, "Dispersion behavior and thermal conductivity characteristics of $\mathrm{Al}_{2} \mathrm{O}_{3}$ $\mathrm{H}_{2} \mathrm{O}$ nanofluids," Current Applied Physics, vol. 9, no. 1, pp. 131$139,2009$.

[8] T. X. Phuoc, M. Massoudi, and R. H. Chen, "Viscosity and thermal conductivity of nanofluids containing multi-walled carbon nanotubes stabilized by chitosan," International Journal of Thermal Sciences, vol. 50, no. 1, pp. 12-18, 2011.

[9] V. Kumaresan and R. Velraj, "Experimental investigation of the thermo-physical properties of water-ethylene glycol mixture based CNT nanofluids," Thermochimica Acta, vol. 545, pp. 180186, 2012.
[10] T.-P. Teng, L. Lin, and C.-C. Yu, "Preparation and characterization of carbon nanofluids by using a revised water-assisted synthesis method," Journal of Nanomaterials, vol. 2013, Article ID 582304, 12 pages, 2013.

[11] Y. Ding, H. Alias, D. Wen, and R. A. Williams, "Heat transfer of aqueous suspensions of carbon nanotubes (CNT nanofluids)," International Journal of Heat and Mass Transfer, vol. 49, no. 1-2, pp. 240-250, 2006.

[12] H. Peng, G. Ding, and H. Hu, "Effect of surfactant additives on nucleate pool boiling heat transfer of refrigerant-based nanofluid," Experimental Thermal and Fluid Science, vol. 35, no. 6, pp. 960-970, 2011.

[13] T. Yousefi, E. Shojaeizadeh, F. Veysi, and S. Zinadini, "An experimental investigation on the effect of $\mathrm{pH}$ variation of MWCNT- $\mathrm{H}_{2} \mathrm{O}$ nanofluid on the efficiency of a flat-plate solar collector," Solar Energy, vol. 86, no. 2, pp. 771-779, 2012.

[14] M. R. Raveshi, A. Keshavarz, M. S. Mojarrad, and S. Amiri, "Experimental investigation of pool boiling heat transfer enhancement of alumina-water-ethylene glycol nanofluids," Experimental Thermal and Fluid Science, vol. 44, pp. 805-814, 2013.

[15] X. Li, D. Zhu, and X. Wang, "Evaluation on dispersion behavior of the aqueous copper nano-suspensions," Journal of Colloid and Interface Science, vol. 310, no. 2, pp. 456-463, 2007.

[16] H.-M. Nieh, T.-P. Teng, and C.-C. Yu, "Enhanced heat dissipation of a radiator using oxide nano-coolant," International Journal of Thermal Sciences, vol. 77, pp. 252-261, 2014.

[17] X. F. Li, D. S. Zhu, X. J. Wang, N. Wang, J. W. Gao, and H. $\mathrm{Li}$, "Thermal conductivity enhancement dependent $\mathrm{pH}$ and chemical surfactant for $\mathrm{Cu}-\mathrm{H}_{2} \mathrm{O}$ nanofluids," Thermochimica Acta, vol. 469, no. 1-2, pp. 98-103, 2008.

[18] Y. J. Hwang, Y. C. Ahn, H. S. Shin et al., "Investigation on characteristics of thermal conductivity enhancement of nanofluids," Current Applied Physics, vol. 6, no. 6, pp. 1068-1071, 2006.

[19] C. J. Ho, L. C. Wei, and Z. W. Li, "An experimental investigation of forced convective cooling performance of a microchannel heat sink with $\mathrm{Al}_{2} \mathrm{O}_{3}$ /water nanofluid," Applied Thermal Engineering, vol. 30, no. 2-3, pp. 96-103, 2010.

[20] R. Kathiravan, R. Kumar, A. Gupta, and R. Chandra, "Preparation and pool boiling characteristics of copper nanofluids over a flat plate heater," International Journal of Heat and Mass Transfer, vol. 53, no. 9-10, pp. 1673-1681, 2010.

[21] T. Yousefi, F. Veysi, E. Shojaeizadeh, and S. Zinadini, "An experimental investigation on the effect of $\mathrm{Al}_{2} \mathrm{O}_{3}-\mathrm{H}_{2} \mathrm{O}$ nanofluid on the efficiency of flat-plate solar collectors," Renewable Energy, vol. 39, no. 1, pp. 293-298, 2012.

[22] M. D. Byrne, R. A. Hart, and A. K. da Silva, "Experimental thermal-hydraulic evaluation of $\mathrm{CuO}$ nanofluids in microchannels at various concentrations with and without suspension enhancers," International Journal of Heat and Mass Transfer, vol. 55, no. 9-10, pp. 2684-2691, 2012.

[23] H. Wang, W. Zhou, D. L. Ho et al., "Dispersing single-walled carbon nanotubes with surfactants: a small angle neutron scattering study," Nano Letters, vol. 4, no. 9, pp. 1789-1793, 2004.

[24] J. Dong, S. Chen, D. S. Corti et al., "Effect of Triton X-100 on the stability of aqueous dispersions of copper phthalocyanine pigment nanoparticles," Journal of Colloid and Interface Science, vol. 362, no. 1, pp. 33-41, 2011.

[25] R. Greenwood and K. Kendall, "Selection of suitable dispersants for aqueous suspensions of zirconia and titania powders using 
acoustophoresis," Journal of the European Ceramic Society, vol. 19, no. 4, pp. 479-488, 1999.

[26] D. Hanaor, M. Michelazzi, C. Leonelli, and C. C. Sorrell, "The effects of carboxylic acids on the aqueous dispersion and electrophoretic deposition of $\mathrm{ZrO}_{2}$," Journal of the European Ceramic Society, vol. 32, no. 1, pp. 235-244, 2012.

[27] Cheap tubes Inc, http://www.cheaptubes.com/default.htm.

[28] T. P. Teng, C. M. Cheng, and C. P. Cheng, "Performance assessment of heat storage by phase change materials containing MWCNTs and graphite," Applied Thermal Engineering, vol. 50, no. 1, pp. 637-644, 2013.

[29] T.-P. Teng and C.-C. Yu, "Heat dissipation performance of MWCNTs nano-coolant for vehicle," Experimental Thermal and Fluid Science, vol. 49, pp. 22-30, 2013.

[30] ASHRAE, "Physical properties of secondary coolants (brines)," in 2009 ASHRAE Handbook-Fundamentals (SI), chapter 31, ASHRAE, 2009. 

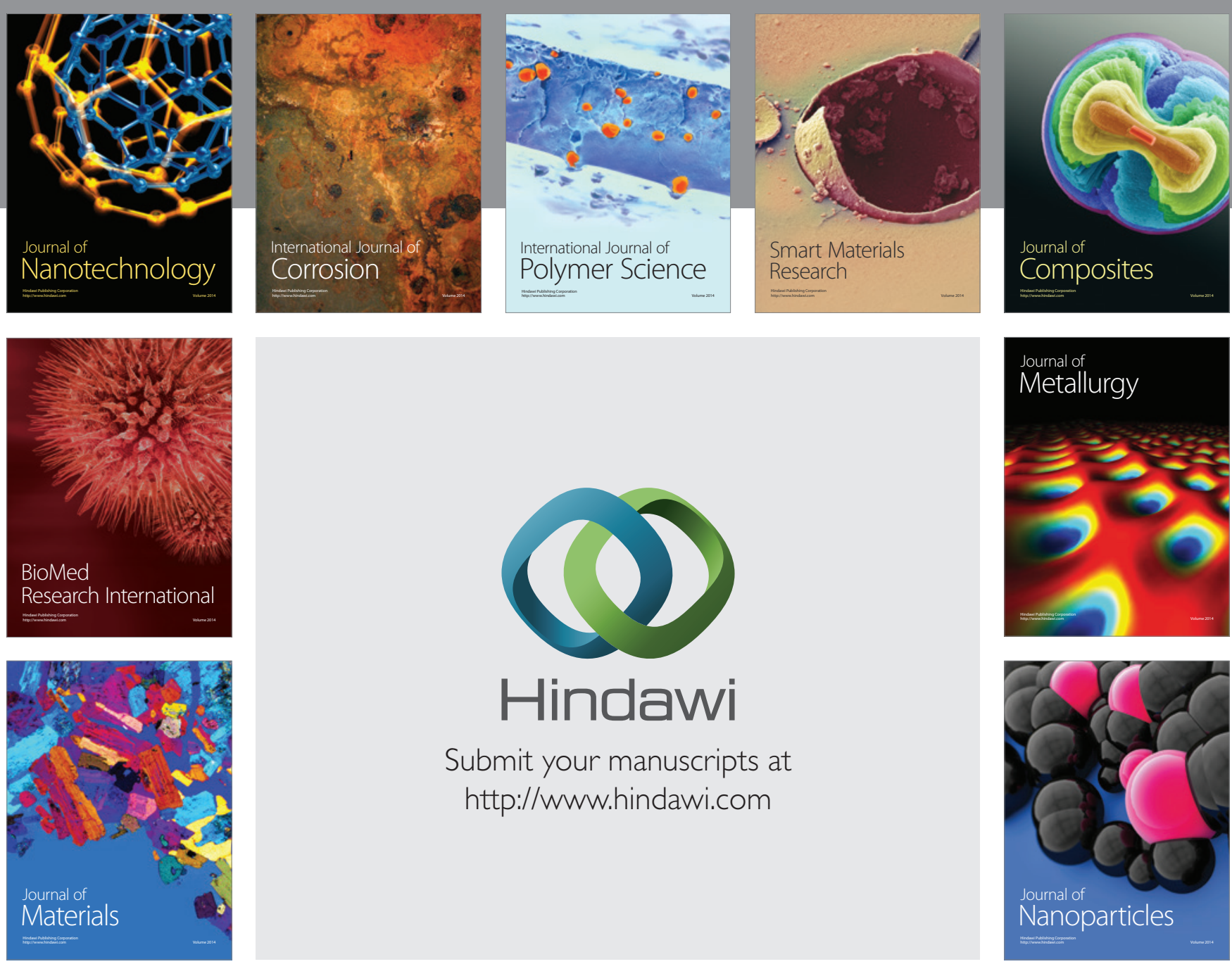

Submit your manuscripts at http://www.hindawi.com
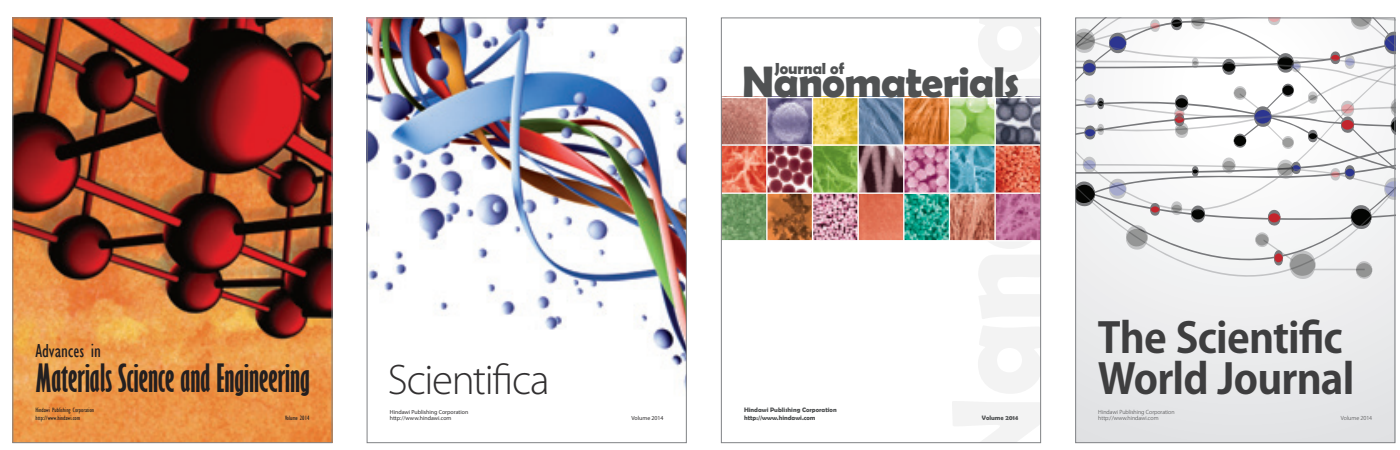

\section{The Scientific World Journal}
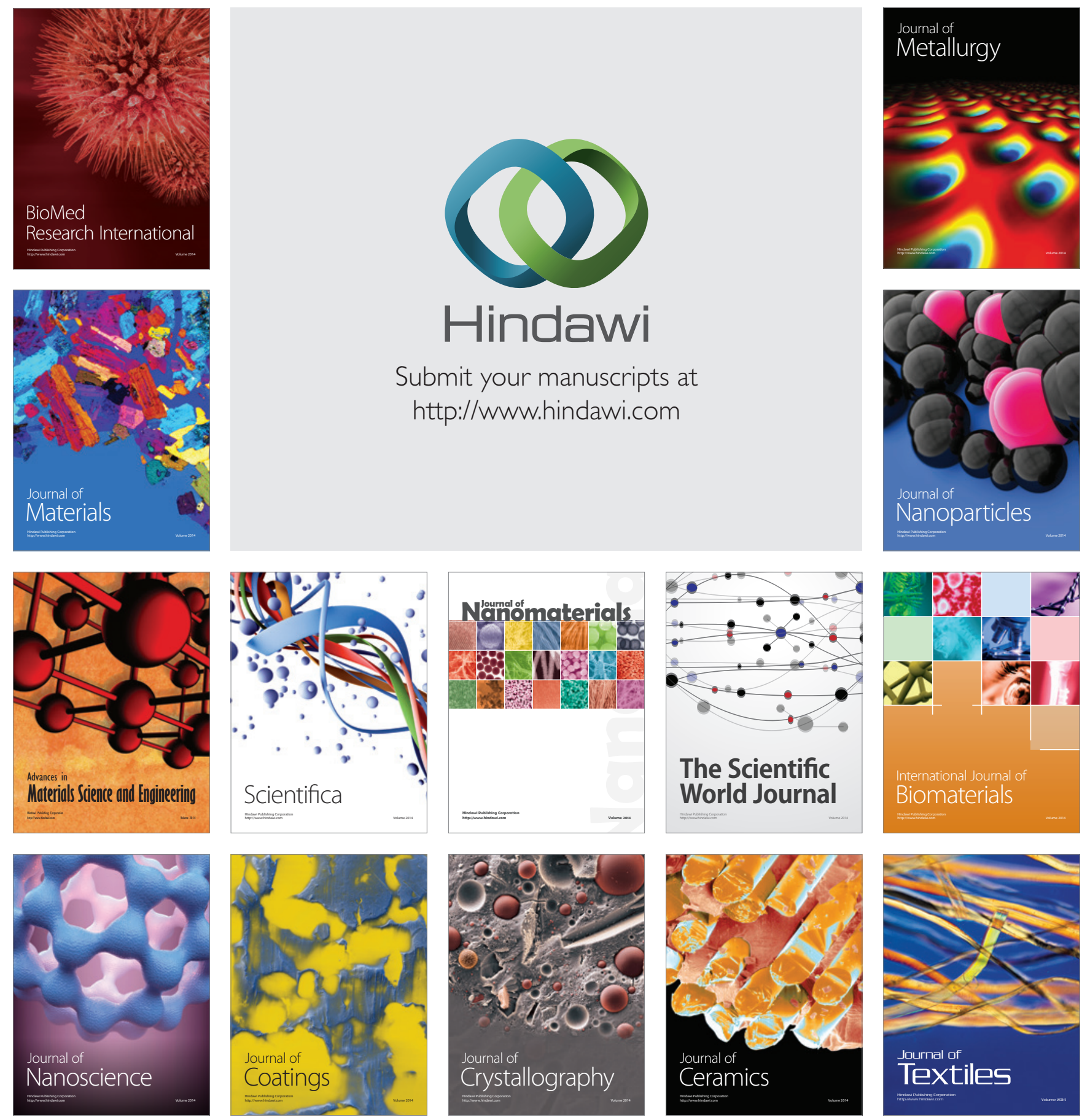